\title{
Antonio Pulido, historia viva de la economía aplicada en España
}

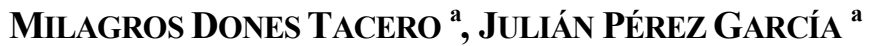 \\ a Universidad Autónoma de Madrid, Fctad. de CC.EE., c/ Francisco Tomás y Valiente , 5, 28049 \\ Madrid,España.E-mail: milagros.dones@uam.es, julian.perez@uam.es
}

\section{RESUMEN}

El objetivo fundamental del presente artículo es el de realizar una revisión de la extensa obra publicada por Antonio Pulido a lo largo de su dilatada carrera docente e investigadora, con el fin de utilizar esta revisión para ilustrar la propia evolución en nuestro país de la economía aplicada, en general, y de la econometría en particular. Para facilitar el seguimiento de una obra tan extensa se han agrupado las aportaciones en torno a siete bloques, que se corresponden con cada uno de los epígrafes del presente artículo y que están vinculados, respectivamente, con la metodología econométrica, modelos aplicados, economía regional, Input-output, Universidad e I+D+i, análisis y prospectiva económica y otras áreas de interés.

Palabras clave: Modelos econométricos, econometría aplicada, Economía regional, Input-Output, Universidad, $\mathrm{I}+\mathrm{D}+\mathrm{i}$, predicción y prospectiva económica.

\section{Antonio Pulido and the Spanish Applied Economy}

\begin{abstract}
Our main objective is to review the extensive work published by Antonio Pulido throughout his long teaching and research career, using this review to illustrate the evolution in our country of applied economics, in general, and econometrics in particular. In order to ease the tracking of such a large work, the contributions have been grouped around seven blocks, which correspond to each of the sections of this article, and which are linked, respectively, with econometric methodology, applied models, regional economy, Input-output, the university and R \& D, the analysis and economic prospects and other areas of interest.
\end{abstract}

Keywords: Econometric Models, Applied Econometrics, Regional Economics, Input-Output, University, R \& D, Forecasting and Economic Prospects.

Clasificación JEL: B31, C10, C20

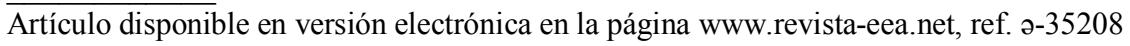




\section{INTRODUCCIÓN}

El objetivo del presente artículo es de realizar una revisión y recopilación de las principales aportaciones que ha realizado el profesor Antonio Pulido San Román, a lo largo de una extensa carrera universitaria que se inició en el curso académico de 1961/62 como profesor ayudante en la Universidad Complutense de Madrid, y que continua en la actualidad, más de 55 años después, como Catedrático Emérito de "Econometría y Métodos estadísticos" en la Universidad Autónoma de Madrid.

Nuestra intención inicial, más allá de rendir nuestro homenaje personal como discípulos directos del profesor Pulido, es la de utilizar esta revisión para ilustrar la propia evolución en nuestro país de la economía aplicada, en general, y de la econometría en particular.

En este sentido, teniendo en cuenta que la econometría, como disciplina formalizada, podemos considerar que se inició a principios del pasado siglo XX y que las primeras aplicaciones puramente econométricas se realizaron en nuestro país entre 1940 y 1970, Castañeda (1945), Echenique (1956), Alcaide (1957), Pena (1960), García Barbancho (1961), Sagristá (1961), Martin Blanco (1962), la trayectoria econométrica de Antonio Pulido, que se inició en 1963 con un su tesis doctoral sobre el análisis econométrico del turismo, puede recoger perfectamente la evolución de esta disciplina en nuestro país, prácticamente desde sus orígenes.

Ahora bien, tal como detallaremos a lo largo del artículo, las aportaciones de este autor han trascendido del campo puramente econométrico y se han extendido a lo largo de todo el espectro que recorre lo que habitualmente denominamos economía aplicada.

Si bien su trayectoria personal le ha mantenido permanentemente vinculado con la actividad universitaria, la consideración de que el conocimiento y la investigación desarrollada en esta institución necesariamente debían estar vinculados con la sociedad le ha llevado a desarrollar una perspectiva personal en la que la transferencia del conocimiento ha guiado la esencia de sus investigaciones.

Es decir, desde un principio ha considerado de vital importancia la conexión entre la universidad, las empresas y las administraciones públicas, habiendo estado involucrado profesionalmente en todas ellas, tal como se refleja en la reseña biográfica que se incluye al final del presente artículo, y siendo uno de los pioneros en el establecimiento de fuertes conexiones entre la investigación universitaria y su entorno socio-económico ${ }^{1}$.

\footnotetext{
${ }^{1}$ En 1981, promovió la creación de Centro de Predicción Económica (CEPREDE) cuyo objetivo fundamental era el de trasladar a las empresas y la administraciones públicas los resultados de la investigación, el análisis y predicción de la economía española realizada desde la Universidad
} 
Con el fin de sistematizar las múltiples aportaciones realizadas por el profesor Pulido, y tratando de huir de un mero relato cronológico de las mismas, hemos tratado de estructurar su obra en torno a siete grandes bloques, que aun teniendo una delimitación un tanto difusa, en cuanto a que la clasificación de algunas aportaciones podría abarcar varios de ellos, consideramos que es representativa de su trayectoria investigadora.

En el primero de los bloques, que se corresponden con los sucesivos epígrafes del presente artículo, hemos tratado de englobar todas aportaciones realizadas en el campo de la metodología econométrica, tanto desde el punto de vista investigador, como docente.

En el segundo bloque hemos incorporado aquellas referencias vinculadas con el desarrollo de modelos aplicados, tanto a nivel agregado o macroeconómico, como a nivel sectorial o microeconómico, dejando todas las aportaciones relacionadas con el análisis regional para un tercer bloque dedicado específicamente a esta línea de trabajo. De la misma forma, todas las aportaciones más vinculadas con la metodología input-output, se han agrupado en un cuarto bloque.

Tal como decíamos, la trayectoria profesional de Antonio Pulido ha estado vinculada permanentemente a la Universidad, siendo la propia organización universitaria, y el papel de ésta en el sistema de $\mathrm{I}+\mathrm{D}+\mathrm{i}$, una de las preocupaciones permanentes a lo largo de toda su carrera. Por este motivo, en el quinto bloque hemos tratado de recopilar todas las aportaciones realizadas por el autor, tanto en el campo de la investigación, el desarrollo y la innovación en términos generales, como en el papel que deben desarrollar en el mismo las universidades, así como su misión general, organización y gobernanza.

Más allá de la propia metodología econométrica y sus aplicaciones en los diferentes campos, la obra de Antonio Pulido es especialmente extensa en términos de lo que podemos considerar como análisis y prospectiva económica en su sentido más amplio, incluyendo, tanto valoraciones permanentes de la situación actual y perspectivas económicas, como análisis de las circunstancias y problemas específicos que ha ido atravesando nuestra economía a lo largo de los últimos años, realizando un esfuerzo especial de divulgación de estos análisis. Por este motivo hemos dedicado un apartado específico para recoger las aportaciones más relevantes en esta área.

Finalmente, cerraremos este artículo incorporando un apartado de miscelánea, donde se ha incorporado otras aportaciones del autor que no son fácilmente englobables en los apartados precedentes.

Autónoma de Madrid y que unos años más tarde, 1991, facilitó la creación del Instituto Universitario de Investigación "L.R.Klein", instituciones que se mantienen activas hasta la actualidad. 


\section{MÉTODOS ESTADISTICO ECONOMÉTRICOS}

El seguimiento de las diferentes publicaciones metodológicas elaboradas por Antonio Pulido a lo largo de su dilatada carrera académica nos ofrece una cierta panorámica de la propia evolución de la disciplina econométrica, en particular, y del análisis económico cuantitativo, en términos generales.

Así, durante sus primeros años, se interesó especialmente por el papel de la econometría como herramienta al servicio del análisis económico Pulido (1969:2,1975:1, 1977:1).

Posteriormente, se centró en los grandes modelos causales multiecuacionales, sus posibilidades, potencialidades y problemática Pulido (1971:1, 1986:1, 1986:2) o Pulido y Fernández (1990:1).

Al final de la década de los ochenta y principios de los noventa, y una vez comprobadas las limitaciones que ofrecían estos grandes modelos para el seguimiento de la coyuntura económica, o la realización de simulaciones simples y operativas, su interés se volvió hacia los modelos más simplificados. Pulido, M.T. y A. Pulido (1992) y las técnicas de análisis de coyuntura y elaboración de indicadores sintéticos. Pulido (1987:1) y Pulido y otros (1989:1).

Ya en los albores del nuevo siglo XXI, la profusión de nuevos desarrollos y planteamientos econométricos alternativos fueron objeto de revisión en Pulido y Pérez (2000 y 2006:1).

En los años más recientes, su interés por los desarrollos metodológicos se ha derivado hacia la elaboración de herramientas informáticas de síntesis información (Business Intelligence, dashboarding), Pulido (2012:1), y el análisis y la medición de la incertidumbre y los riesgos económicos Pulido (2016:1).

Analizando con una cierta perspectiva las múltiples aportaciones realizadas por Antonio Pulido en el ámbito de la metodología estadístico-econométrica, podemos identificar tres grandes líneas o denominadores comunes que han estado presentes a lo largo de toda su obra.

En primer lugar, es fácil identificar que la mayoría de sus obras, sin perjuicio del rigor metodológico, tienen un carácter inminentemente aplicado, siendo, precisamente, la búsqueda de la utilidad final y la aplicabilidad de las diferentes metodologías el objetivo básico perseguido por el autor.

Directamente vinculada con esta aplicabilidad estaría la segunda de las características generales de estas aportaciones y que podríamos identificar como el especial interés que muestra por la transferibilidad de estas técnicas haciéndolas accesibles, tanto a los alumnos como a los profesionales del análisis económico, y tratando de alejarlas de las "torres de marfil" en las que estaban situadas, sobre todo en las etapas iniciales de su desarrollo y donde sólo unos pocos "iniciados" parecían ser capaces de desarrollar estos modelos. 
Finalmente, un tercer denominador común lo encontramos en el enfoque predictivo o prospectivo, sobre el que están focalizadas una buena parte de las aportaciones revisadas.

\subsection{Estadística y econometría aplicada}

El carácter fundamentalmente aplicado de la obra de Antonio Pulido está presente desde los inicios de su trayectoria profesional siendo una de sus primeras aportaciones, precisamente, la aplicación de la metodología econométrica al turismo, objeto de su tesis doctoral y que fue publicada posteriormente por el Instituto de Estudios Turísticos Pulido (1966:1). En la misma línea, unos años más tarde publica un nuevo libro de metodología econométrica, aplicada, en este caso, a la inversión. Pulido (1974:1).

A finales de los años sesenta la incipiente difusión de las herramientas informáticas abrió toda una ventana de posibilidades de desarrollar aplicaciones econométricas y así lo recogía en un documento de trabajo del centro de cálculo de la universidad de Madrid. Pulido (1969:1).

A medida que se iban realizando diferentes aplicaciones y desarrollos econométricos aumento el interés del autor por transmitir a la sociedad en general y a las empresas en particular la potencialidad que tenían estas herramientas en las tareas de gestión y planificación económica y así se pone de manifiesto en diferentes conferencias, tales como "Experiencia sobre utilización de modelos econométricos en la política económica española" impartida en 1972 en el "Seminario Internacional de Modelos Matemáticos aplicados a la Enseñanza", Ministerio de Educación y Ciencia, o en la 16 Conference on Industrial Business and Marketing Opportunities for the Enlarged Common Market bajo el título "Una poderosa herramienta de predicción para la planificación estratégica: modelos macroconométricos" EVAF, 1981; así como en múltiples artículos Pulido (1978:1, 1980:1, 1983:2,1988:1,1993) y documentos de trabajo Pulido y otros (1979:1, 1989:2).

A pesar de los múltiples avances realizados y de la multiplicación de las diferentes técnicas estadístico-econométricas de los últimos años, esta preocupación por la aplicabilidad de las mismas ha seguido presente en su obra tal como se recoge en diversos artículos como Pulido (2002:1) o Pulido (2005:1)

\subsection{Transmisión de técnicas estadístico-econométricas}

Tal como adelantábamos, el interés del profesor Pulido por la enseñanza y la transmisión de la metodología y las aplicaciones de las técnicas cuantitativas a la economía ha sido otro de los elementos constantes a lo largo de su trayectoria profesional, y así se refleja en aportaciones como "Algunas iniciativas pedagógicas en la enseñanza de la economía" Pulido (1987:2), o "La enseñanza de economía aplicada asistida por ordenador" Pulido (1990:1). 
En cualquier caso, son sus materiales docentes el mejor ejemplo de este interés por la transmisión de conocimientos, desde de sus primeras aportaciones en "Estadística y técnicas de investigación social" Pulido (1967:1), editado por primera vez en 1967 y reditado en varias ocasiones hasta principios de los años 80, o en econometría, junto con Angel Alcaide en la UNED. Pulido y Alcaide (1977); aunque es, sin duda, su manual sobre Modelos Econométricos Pulido (1983:1) el principal exponente de esta vocación docente.

En este manual se realiza una revisión rigurosa y sistemática de los principios básicos de la econometría en su sentido más amplio, desde el planteamiento de los modelos hasta su utilización, pasando por las técnicas de estimación, contrastación y validación de modelo, prestando especial atención a los desarrollos más evolucionados de los modelos estructurales multiecuacionales.

Sin embargo, posiblemente la característica más diferenciadora de este manual en el momento de su publicación fue la incorporación de múltiples ejemplos prácticos y aplicaciones reales de todo tipo de técnicas, reflejando esa impronta de "económetra aplicado" que destacábamos en el apartado anterior, y que se amplió aún más en la versión renovada del manual publicada en 2001, Pulido, A. y J. Pérez (2001).

Este mismo carácter aplicado se refleja igualmente en otro de sus manuales de referencia, realizado en colaboración con el profesor Santo Peña, dedicado a la estadística aplicada y especialmente pensado para que el texto sea útil en una enseñanza práctica de la estadística a través de los ordenadores personales. Pulido, A. y J. Santos (1998).

\subsection{Metodología y técnicas de predicción}

El tercero de los denominadores comunes lo encontramos en el enfoque predictivo o prospectivo, que ha dominado una buena parte de la trayectoria, tanto docente, como investigadora, de Antonio Pulido.

En esta línea, al autor ha elaborado diferentes artículos y documentos de trabajo, dedicados al metodologías específicas de predicción, desde las aproximaciones más sencillas Pulido (1971:2,1988:2), hasta las más avanzadas Pulido (1982:1, 1988:3, 1992:1), culminando con la primera edición en el año 1989 con la publicación de su libro "Predicción Económica y Empresarial" Pulido (1989:1) donde realiza un compendio práctico de múltiples técnicas aplicadas de predicción del que, diez años más tarde y en colaboración con la profesora Ana López, realizó una segunda versión, con un enfoque mucho más práctico, si cabe, y que incorporaba cerca de un centenar de ejemplos y once casos reales completos que el lector puede, además, reproducir y analizar en su propio ordenador personal con programas de tan alta difusión como Excel y EViews, incluyendo como principal novedad un simulador de la economía española (modelo MICRO-UAM). 
En esta obra se recogen desde las técnicas más simples de predicción y simulación a las más profesionalizadas, tanto para el tratamiento de datos cuantitativos como de información subjetiva, incluyendo la aplicación de ajustes de tendencia, desestacionalización, encuestas, Delfos, alisados exponenciales, modelos ARIMA, VAR y cointegración, modelos econométricos y otros enfoques diversos. Pulido y López (1999).

Al margen de la propia metodología y técnicas de predicción, el autor ha dedicado también una buena parte de su trabajo a la reflexión sobre la actividad de predicción y la prospectiva, su papel en el proceso de análisis y toma de decisiones, así como a la propia tarea de divulgación de la actividad predictiva y un buen ejemplo de ello lo podemos encontrar en Pulido y otros (1977:1) o Pulido (1983:3, 1988:4, 2006:1, 2007:1, 2013).

\section{MODELOS APLICADOS}

Tal como hemos señalado en los apartados precedentes el planteamiento y desarrollo de modelos aplicados ha sido una constante a lo largo de la trayectoria profesional de Antonio Pulido de forma tal que una buena parte de sus contribuciones se concentran en la presentación de diferentes aplicaciones econométricas.

Nuevamente con un ánimo de simplificar al lector el seguimiento de estas aportaciones hemos considerado oportuno agruparlas en torno a tres grandes bloques, aplicaciones macroeconómicas agregadas, grandes modelos y aplicaciones sectoriales; si bien, como en otras ocasiones, esta clasificación tiene un carácter ilustrativo y no excluyente ya que la asignación a uno u otro bloque tiene un alto grado de subjetividad.

\subsection{Aplicaciones macroeconómicas}

Dentro de este primer grupo hemos recogido aquellas aplicaciones que teniendo un carácter macroeconómico, no están directamente vinculadas con la construcción de grandes modelos y que se presentarán en al apartado siguiente.

Generalmente, estas aplicaciones están concentradas en el inicio de la carrera investigadora de Antonio Pulido, y reflejan, de alguna forma, el interés que existía en ese periodo por los temas vinculados a una economía que estaba en plena etapa de desarrollo.

Así, estas primeras aportaciones están dedicadas al propio desarrollo económico, Pulido (1966:2), el desempleo, Pulido (1966:3), la educación, Pulido (1966:4), o las funciones de producción, Pulido (1968:1).

Unas décadas más tarde, estas aplicaciones se centraron en los problemas del desempleo, Pulido (1978:2), Pulido y Pena (1986), y la inflación, Pulido (1989:2). 


\subsection{Grandes modelos}

Si bien la participación de Antonio Pulido en la elaboración de grandes modelos se inició en los años 70 con uno de los primeros modelos agregados desarrollados para la economía española, el COPLAN ${ }^{2}$, Pulido y otros (1971) y ha estado involucrado en diversos proyectos de integración internacional de modelos como el proyecto LINK de Naciones Unidas, Pulido (1982:2) Pulido y otros (1982:1) o el Proyecto HERMES de la Comisión Europea Pulido y otros (1983:1, 1983:2), es, sin duda, el lanzamiento y la dirección del Modelo Wharton-UAM, la aportación más significativa de este autor.

Tal como se recoge en Pulido y Pérez (2001) el Modelo Wharton-UAM es un modelo de análisis y previsión de la economía española que se viene desarrollando desde hace más de 30 años, en el seno del Instituto de Predicción Económica "L.R.Klein" de la Universidad Autónoma de Madrid, y que, desde mediados de los años ochenta se utiliza de forma permanente para ofrecer predicciones y asesoramiento macroeconómico a un conjunto de más de treinta empresas e instituciones públicas y privadas que forma parte de la Asociación Centro de Predicción Económica CEPREDE.

Desde su inicio, el modelo tenía establecidos unos objetivos básicos que podemos resumir en su capacidad para predecir la evolución de la economía española con un horizonte de medio y largo plazo, con la desagregación suficiente como para cubrir las necesidades de información sobre el futuro, a nivel macroeconómico, de las instituciones, públicas o privadas, anteriormente citadas, y permitir la simulación de políticas alterativas relevantes y escenarios alternativos de evolución de la economía internacional.

La especificación inicial de este modelo, con datos hasta 1977, fue realizada aprovechando la experiencia de Wharton en modelos similares y los trabajos iniciales de J.M.Viaene (1980). A partir de esta especificación se procedió a una revisión y actualización de la base de datos, a fin de disponer de series homogéneas de 1954 a 1978, Martínez (1979). La primera versión del modelo Wharton-UAM como tal, fue bautizada como Modelo España-Cero, y está recogida en Vicéns (1979), mientras que las características fundamentales de estos planteamientos iniciales pueden encontrase en Pulido (1981:1), Pulido y otros (1982:2) y Pulido y Sur (1986:1).

Son múltiples los documentos de trabajo y artículos que se han generado a lo largo del proceso de construcción de este modelo, abordando diversos aspectos del mismo como la modelización de los tipos de cambio, Pulido, Fernández y

\footnotetext{
${ }^{2}$ En este modelo desarrollado a principios de los años setenta se integraron, junto a Antonio Pulido, un amplio equipo de investigadores entre los que se encontraban J. Alcaide Guindo, A. Alcaide Inchausti, J.R. Alvarez Rendueles, J.A. Gallego Gredilla, J.D. García Martínez, J.L. del Hoyo Bernat, A. Maurín Flores y A. Moral Muñoz.
} 
Fontela (1987); los tipos de interés, Pulido (1989:3); los precios y salarios, Pulido y Castilla (1983), Pulido, Fernández y Vicéns (1988); el ahorro, la financiación y la inversión, Pulido (1983:4 y1983:5); el sector exterior, Pulido y otros (1983:3); el consumo, Pulido y otros (1982:3); la producción, Pulido y otros (1982:4); o el tratamiento del déficit y la deuda pública, Pulido y Pérez (1992).

De la misma forma, se han generado diferentes publicaciones recogiendo resultados de predicción Pulido y Castilla (1982), Pulido (1984:1 y 1984:2), Pulido y García (1986), o simulaciones realizadas con el modelo sobre los efectos del tipo de cambio, Pulido y Barbero (1984), o sobre el sector exterior, Pulido y Sur (1986:2), Pulido y Fernández (1986).

\subsection{Aplicaciones sectoriales}

Junto a estos grandes modelos, la trayectoria profesional de Antonio Pulido le ha llevado a analizar, con mayor menor nivel de profundidad, diferentes ramas de actividad o sectores productivos, mediante aproximaciones econométricas.

Así, al inicio de su carrera realizó diferentes aplicaciones en política laboral, Pulido y otros (1967:1), Pulido (1969:3); turismo, Pulido (1965, 1966:5, 1967:2, 1968:2, 1979:1) Pulido y otros (1965, 1967:2, 1975); el sector comercial y de distribución, Pulido (1976:1), Pulido y otros (1976, 1977:2, 1977:3,1979:2); el modelo educativo, Pulido(1971:3), Pulido y otros (1969); la actividad del sector público, Pulido y otros (1970); o el sector del transporte, Pulido (1972).

Posteriormente, y ya como director del Instituto de Predicción Económica "L.R.Klein" ha participado en diversos proyectos de análisis y predicción sectorial entre los que cabría destacar el del sector de las telecomunicaciones, Pulido y Vicéns (1990), el sector de automoción, Pulido y Fernández (1990:2), las actividades de construcción, Pulido y López (1993), Pulido y Mur (1993); y más recientemente, la actividad aseguradora en el contexto de la Cátedra Mapfre-UAM, Pulido y otros (2014).

\section{ECONOMÍA REGIONAL}

Los análisis y modelos regionales han sido otra de las grandes líneas de investigación desarrolladas por Antonio Pulido y en la que podemos ubicar algunas de sus grandes contribuciones.

Así, tras los trabajos iniciales sobre desarrollo y modelización regional en la década de los 70 y los 80, Pulido (1973:1, 1980:2, 1984:3) y Pulido y otros (1974), a finales de los ochenta, dirige junto el profesor Bernardo Pena, el lanzamiento del proyecto HISPALINK de modelización regional integrada y que trata de replicar la experiencia internacional del Proyecto LINK de Naciones Unidas pero a escala nacional. 
Tal como recogen Pulido y Cabrer (1994) el Proyecto Hispalink constituye una línea de investigación en economía aplicada de un conjunto de universidades españolas. Su objetivo es la revisión y mejora permanente del análisis de la situación y perspectivas económicas de las regiones españolas. Para alcanzar tales objetivos, cada equipo ha desarrollado un modelo econométrico que trata de describir con suficiente aproximación el comportamiento de la región correspondiente. El conjunto de equipos dispone de una base de datos en común, utiliza las mismas predicciones iniciales para el conjunto de la economía española y coordina las predicciones regionales a través de un modelo de congruencia. Los resultados del Proyecto Hispalink se ponen a disposición de la sociedad española a través de las instituciones nacionales y regionales que patrocinan el proyecto.

Alrededor de este proyecto se ha generado diversas contribuciones del autor, desde los planteamientos iniciales Pulido (1990:2, 1991:1, 1992:2), Pulido y Fontela (1992), Pulido, Fontela y Sur (1990:1, 1990:2), pasando por la construcción de la base de datos HISPADAT, Pulido, López y Rivero (1996), hasta la explotación de los resultados del proyecto, Pulido (1992:3,2001:1), Pulido y Callealta (1994).

Al margen de los trabajos realizados en el seno del mencionado proyecto Hispalink, el profesor Pulido ha realizado otras investigaciones en el ámbito regional, vinculadas con los procesos de integración regional Pulido (1995:1), las consecuencias regionales de la ampliación europea, Pulido, López y Castro (2003), en análisis de las cadenas regionales de producción Pulido y López (2002:1), estimación de contabilidad regional trimestral, Pulido, Castro y López (2003) y Pulido y otros (2001:1), evaluación regional de la economía digital, Pulido y López (2001:2), o planteamientos y reflexiones generales sobre el análisis regional, Pulido (1988:5, 1995:1, 1995:2, 2009:1).

Adicionalmente, el autor ha realizado diversos trabajos, no ya de tipo multirregional como los comentados en los párrafos precedentes, sino focalizados en una única comunidad.

Así, en el ámbito de la Comunidad de Madrid, ha realizado diversos trabajos de modelización y previsión de la economía regional, Pulido y Sur (1987), Dones y otros (2005), Pulido y Ros (2002, 2005), análisis de comercio exterior regional, Llano y otros (2001), Pulido y otros (2003), el sector financiero en la región, Pulido (2005:2), o la propia dinámica de crecimiento regional Lopez y Pulido (2003:1).

Igualmente ha realizado diversos análisis y proyecciones de la economía local de la ciudad de Madrid, elaborando desde el año 2006 hasta la actualidad el capítulo del entorno económico del informe de "Situación y perspectivas económicas de la Ciudad de Madrid" que publica semestralmente el observatorio económico del Ayuntamiento de Madrid y ha colaborado en el informe "Madrid en el concierto de las grandes ciudades europeas y mundiales" publicado en 2005 
por la misma institución, con un capítulo dedicado a la visión estratégica y otro a los indicadores para un análisis estratégico; este último vinculado al proyecto URBAN AUDIT que el INE realizó en colaboración con el Instituto "L.R.Klein" bajo la dirección de Antonio Pulido y Ana López.

Para la Comunidad Autónoma de Andalucía, también ha realizado diversos trabajos sobre el comercio exterior Llano y Pulido (2002), Pulido (2010); la nueva economía, López y Pulido (2003:2), los condicionantes externos, Pulido (1994:1); o los efectos de la deslocalización (dirección del equipo de trabajo del proyecto elaborado para la Agencia de Innovación y Desarrollo de Andalucía. IDEA).

\section{ANÁLISIS INPUT-OUPUT}

Al igual que los análisis regionales, el desarrollo de modelos y aplicaciones basados en las tablas Input-Output has sido otra de las constantes a lo largo de la trayectoria académica de Antonio Pulido desde sus inicios, Pulido, García y Cortiñas (1964); habiendo realizado tanto contribuciones de carácter metodológico, como de creación de tablas input-output y, por supuesto, múltiples trabajos aplicados.

Así, en el campo metodológico, podemos resaltar sus aportaciones en el análisis del cambio tecnológico y su vinculación con el desarrollo económico, Pulido(1986:3), Fontela y Pulido (1986, 1989:1, 19889:2), la integración con los sistemas de cuentas nacionales, Fontela y Pulido (1990:1), la predicción y simulación con modelos input-output, Fontela y Pulido (1992), o la potencialidad de estas aplicaciones y sus posibilidades de desarrollo futuro, Pulido y Fontela (1993:1), Fontela y Pulido (2005), Antille, Fontela y Pulido (2005).

Sin embargo, su aportación más difundida fue el libro publicado en 1993 junto con su gran compañero Emilio Fontela Montes, y donde bajo el título de "Análisis Input-Output. Modelos, datos y aplicaciones" se presenta todo un recopilatorio de metodología y tratamiento de tablas input-output. Pulido y Fontela (1993:2).

El libro está diseñado para que el lector pueda adoptar una posición activa en el proceso de aprendizaje y donde cada capítulo contiene, aparte de ejemplos y cuadros sinópticos, aplicaciones que pueden seguirse en un ordenador personal con un programa de hoja de cálculo. En el mismo, se aborda tanto la amplia problemática de elaboración de tablas y su conexión con los sistemas de cuentas nacionales, como los diversos modelos disponibles, análisis de resultados y aplicaciones.

Entre las aportaciones centradas en las aplicaciones de la metodología inputoutput podemos encontrar, análisis de impactos sectoriales en demanda y precios, Fontela y Pulido (1973); marketing y turismo, Pulido (1976:2); análisis 
de la productividad, Fontela y Pulido (1990:2); contraste de modelos de predicción, Mur y Pulido (1992), o el análisis de las cadenas de producción, Lopez y Pulido (2000).

En esta misma línea de grandes aplicaciones de la metodología input-output, a mediados de los años noventa Antonio Pulido encabezó un grupo de investigación dedicado a la exploración de las posibilidades de elaboración de tablas input-output interregionales para la economía española, Pulido (1995:3), Pulido (1996:1), y para cuya elaboración se hacía necesaria la estimación de los flujos de comercio interregionales.

Como continuación de este proyecto inicial en el año 1998 se firmó un convenio de colaboración con la Dirección de Análisis y Programación Presupuestaria del Ministerio de Economía financiado con fondos FEDER del Programa Operativo de Asistencia Técnica (Objetivo 1) 1994-1999 y cuyo objetivo era la creación de unas tablas input-output interregionales de la economía española que fueron finalmente estimadas para el año 1995 y que se han mantenido actualizadas en años posteriores, Pérez, Llano y García (2009) y que han sido utilizadas para analizar los impactos interregionales de los fondos estructurales y de cohesión Dones, Pérez y Llano (2009).

También como continuidad de este proyecto inicial, y partir de los desarrollos iniciales de la tesis doctoral de Carlos Llano ${ }^{3}$, dirigida por Antonio Pulido en el año 2001, se creó el proyecto C-Intereg, que se mantiene activo hasta la actualidad (www.c-intereg.es) y cuyo objetivo es la estimación continua de los flujos de comercio entre las diferentes regiones españolas. Llano y otros $(2009,2010)$.

\section{UNIVERSIDAD E I+D+I}

Desde que en el año 1961 se incorporara como profesor ayudante en la Universidad Complutense de Madrid y tras su breve paso por la Universidad de Valencia, Antonio Pulido ha desarrollado toda su trayectoria profesional como catedrático a tiempo completo en la Universidad Autónoma de Madrid, donde ha ocupado cargos de dirección y gestión universitaria, tanto a nivel de departamento, como de instituto de investigación, demostrando siempre un elevado interés por la labor docente e investigadora de la universidad, así como su papel en el esquema general de investigación, desarrollo e innovación, tal como demuestran sus múltiples aportaciones en este campo. Pulido (2006:1), Fontela y otros (1990).

\footnotetext{
3 "Economía sectorial y espacial: el comercio interregional en el marco input-output" Tesis doctoral de Carlos Llano Verduras dirigida por Antonio Pulido San Román. Universidad Autónoma de Madrid (2001).
} 
Comenzaremos por realizar una revisión de las aportaciones centradas en la labor y gobernanza universitaria, para posteriormente, presentar aquellas más focalizadas en la $\mathrm{I}+\mathrm{D}+\mathrm{i}$ propiamente dicha.

\subsection{La Universidad: misión y gobernanza}

Las primeras contribuciones publicadas por el autor es este campo se ocupaban de la labor a realizar en las universidades, Pulido (1981:2, 1984:4, 1990:3), Pulido y otros (1973:1), preocupación que ha continuado presente a lo largo de su trayectoria profesional, prestando especial atención a las necesidades de cambio y la prospectiva del futuro universitario, Pulido (1984:5, 1986:4, 2001:2, 2006:2, 2007:2, 2007:3, 2007:4, 2007:5).

Este interés por el futuro de la universidad le llevó a promover, junto con un amplio grupo de profesores universitarios, el foro UNIVNOVA cuyo objetivo básico, Pulido (2007:6), es el de propiciar un foro de discusión abierto en que se analicen enfoques y propuestas alternativas con una visión prospectiva, focalizado en torno a tres tareas concretas:

1. Realizar una prospectiva de futuro de la Universidad en general y de sus posibles variantes en Europa y en España.

2. Detectar los principales factores condicionantes del éxito de las estrategias para adaptar la universidad española a los nuevos requerimientos.

3. Proponer medidas concretas de actuación y sensibilizar a los agentes sociales implicados.

En el contexto de esta iniciativa (www.univnova.org) se encuadran una buena parte de las contribuciones realizadas por Antonio Pulido y que se resumen en su libro "El Futuro de la Universidad", Pulido (2009:2) y cuya intención es hacer reflexionar a políticos, empresarios, líderes sindicales o de instituciones no lucrativas, intelectuales o ciudadanos en general, sobre la universidad del presente y, sobre todo, de la que necesitamos para abordar, con ilusión, un futuro más o menos inmediato. Con esta finalidad se incorporan, en dosis variables a lo largo de los diferentes capítulos, información y opinión. La componente informativa trata de plantear los grandes temas y suministrar el material de base disponible en muy diversas publicaciones. La opinión proviene tanto del propio autor como de la valiosa colaboración de unos entrevistados de lujo: dos Premios Nobel (Richard Ernst y Lawrence Klein), tres rectores (Gregorio Peces-Barba, Ángel Gabilondo, entonces Ministro de Educación y Juan Vázquez); dos empresarios con una visión global (Eduardo Montes y Juan Rodríguez-Inciarte) y tres expertos reconocidos internacionalmente (Francisco Marcellán, Emilio Fontela y José-Ginés Mora).

Adicionalmente a esta preocupación por el funcionamiento actual y las necesidades de futuro de la labor universitaria, la trayectoria docente de Antonio 
Pulido le ha llevado a participar de forma activa en los procesos de valoración y acreditación del profesorado siendo miembro de la Comisión asesora del programa ACADEMIA de la Agencia Nacional de Evaluación de la Calidad y Acreditación (ANECA) y Presidente del Comité de Ciencias Sociales y Jurídicas del Programa de Evaluación del Profesorado de la Agencia Nacional de Evaluación de la Calidad y Acreditación (ANECA), coordinando el subcomité de Ciencias Sociales y Jurídicas entre los años 2006-2007, lo que le llevó a proponer diferentes alternativas de cuantificación de la calidad docente e investigadora del personal universitario, Pulido (2005:3), Pérez y Pulido (2005).

\subsection{Investigación, Desarrollo e Innovación}

Ya a finales de los años ochenta en su libro "El reto de la investigación para la empresa" ponía de manifiesto la problemática de la investigación en nuestro país y la inadecuada vinculación entre las universidades, las empresas y las administraciones públicas. "Una política científica que ha desatendido la investigación, cuando el desarrollo de ésta debería haber antecedido a la política industrial, así como el equivocado papel que se ha hecho jugar a la universidad, constituye la simiente provocadora de esta situación. Se hace necesario el cambio de mentalidad, el de la universidad para responder al reto de la investigación de una sociedad moderna, y el de la empresa, especialmente la mediana y pequeña, que se encuentran totalmente desasistida, sin recursos humanos y materiales suficientes, recursos que pueden encontrar fácilmente en la universidad en condiciones óptimas", Pulido (1979:2).

Esta necesaria vinculación entre las universidades, las empresas y las administraciones en el desarrollo de las actividades de $\mathrm{I}+\mathrm{D}+\mathrm{i}$, ha sido un denominador común en la mayoría de las aportaciones publicadas por el autor en esta materia. Pulido (1982:4, 1982:5, 2008:1, 2008:2), Pulido y Fontela (2008), Pulido y otros (1980:1, 1981:1), Fontela y otros (1992).

Así, en su libro "Investigación Innovadora" señala que, "por unos u otros motivos, ni la Investigación ocupa en España el lugar que le correspondería por el nivel alcanzado de desarrollo económico, ni la interacción UniversidadEmpresa en Investigación ha sobrepasado los límites de lo anecdótico" , resaltando que "una parcela de especial interés es la de la Investigación que conjuntamente puedan desarrollar Empresas y Centros Universitarios, en el contexto de los Planes de Ciencia y Tecnología que pueda lanzar la Administración de nuestro país en los próximos años". Pulido y otros (1981:2).

De la misma forma, la preocupación del autor siempre ha estado vinculada con los efectos finales de la investigación, el desarrollo y la innovación sobre el conjunto del sistema económico, en general, y especialmente sobre la productividad y competitividad, Pulido (1983:6, 1983:7, 2004:1, 2004:2, 2005:4, 2005:5). 
En esta línea de análisis de los efectos económicos de la investigación, el desarrollo y la innovación, el autor ha publicado diversos trabajos sobre la economía de los intangibles, Pulido (2008:3, 2008:4, 2009:3), o la biotecnología, Pulido y otros (2007), Dones, Pérez y Pulido (2008, 2010); si bien, es en al ámbito de las Tecnologías de la información y las comunicaciones (TIC) y la "nueva economía", donde se concentran la mayoría de sus contribuciones en esta línea, Pulido (1982:6, 2000:1, 2001:3, 2001:4, 2001:5, 2001:6, 2002:2, 2002:3), Pulido y López (2001:1,2003), López y Pulido (2002, 2003:3) y Pérez y Pulido (2001); habiendo sido el impulsor en el año 2000 del proyecto N-economía, que sigue vigente en la actualidad, y que constituye un punto de referencia de información sobre nueva economía, nuevas tecnologías, innovación, sociedad de la información y del conocimiento; teniendo como objetivo general el promover la información económica sobre las TIC y la innovación en general y sus efectos a escala internacional, de la UE, de la economía española, regional y de sectores de actividad y empresas (http://n-economia.com/).

\section{ANÁLISIS Y PROSPECTIVA ECONÓMICA}

Con independencia de todas las aportaciones metodológicas y aplicadas que hemos ido recogiendo en los apartados anteriores, si hay algo que ha caracterizado la trayectoria profesional de Antonio Pulido ha sido su enorme producción en términos de lo que podemos considerar la economía más aplicada, es decir, el seguimiento permanente de la situación actual y las perspectivas de evolución de la economía mundial, en general, y de la economía española en particular.

Así, al margen de todas las aportaciones que comentaremos a continuación, Antonio Pulido ha venido reflexionando permanentemente sobre las diferentes situaciones, problemas y retos que ha ido enfrentando la economía española a lo largo de los últimos cuarenta años, dejando multitud de páginas escritas en todo tipo de documentos de trabajo, informes, artículos de prensa $\mathrm{y}$, más recientemente, redes sociales, y que sería imposible de registrar de forma sistemática en el contexto del presente artículo.

A modo de ejemplo, baste señalar que en el contexto del proyecto de predicción económica CEPREDE al autor lleva realizando de forma ininterrumpida desde el año 1981 dos informes anuales de situación y perspectivas de la economía española muchos de los cuales están accesibles a través de la página web del citado proyecto (www.ceprede.es); mientras que desde principios del año 2015, hasta el momento actual, lleva acumuladas más de 150 entradas en su blog (http://blog.antoniopulido.es/) y más de 3200 tweets (https://twitter.com/PsrA).

Si bien es cierto que las contribuciones del autor en esta área presentan una tipología muy diversa, existen, como en otros apartados, unas líneas básicas 
sobre las que se agrupan una buena parte de ellas.

Así, por ejemplo, todos los temas vinculados con el crecimiento económico, sus causas y consecuencias han sido objeto de diferentes aportaciones, tales como Pulido (1973:2, 1977:2, 1981:3, 1991:2), Pulido y Pérez (2006:2), Pulido y otros (1973:2), López y Pulido (2005); aunque su obra más extensa se refleja en el libro "Economía en Acción" donde se recogen y analizan más de mil experiencias sobre crecimiento económico y sus causas, desde los análisis clásicos de productividad y contabilidad del crecimiento, a los más modernos de convergencia, condicionantes sociopolíticos o nueva economía. Así mismo, se presenta un resumen en forma de 55 hechos estilizados, o reglas empíricas de funcionamiento, de la economía en acción que el autor complementa con una revisión de opiniones, creencias y métodos para obtener esas leyes empíricas. Finalmente, se incluyen anexos que ayudan a entender las aplicaciones comentadas, así como una selección de más de cien cuadros de resultados de los trabajos de referencia más importantes. Pulido (2000:2).

El posicionamiento de la economía española en el contexto de la economía mundial, especialmente en relación con la Unión Europea y los retos que plantea para las empresas, es otro de los temas que ha sido abordado por el autor en diferentes ocasiones, Pulido (1991:3, 1994:2, 1994:3, 1997:1, 2003:2, 2015:1, 2016:2).

De la misma forma, los temas vinculados con el empleo, Pulido (1981:4), Pulido, Fontela y Lo Cascio (1989), o la política económica, en su sentido más amplio, tampoco han sido olvidados a lo largo de su obra, Pulido (1995:4, 1995:5, 1996:2), Pulido y Fontela (1995), Dones y Pulido (1986).

En cualquier caso, son todos los aspectos vinculados con la prospectiva económica y las predicciones de futuro donde se han acumulado gran parte de los trabajos aplicados desarrollados por el autor a lo largo de toda su trayectoria, Pulido (1980:3. 1981:5, 1983:7, 1983:9, 2001:7, 2001:8, 2004:3, 2006:3, 2007:7, 2008:5, 2009:4, 2012:2, 2016:3), Pulido y Fontela (2002), Pulido, Fontela y Gutiérrez (1987), López y Pulido (2010).

No podemos cerrar este apartado dedicado a las contribuciones realizadas por Antonio Pulido en el ámbito del análisis y la prospectiva económica sin poner de manifiesto el elevado esfuerzo que ha realizado para hacer llegar al público no especializado todo tipo de teorías, conceptos y análisis económicos; y que, sin renunciar al necesario rigor que debe presidir la obra de todo investigador, consigue hacerlos accesibles.

Esta labor de divulgación científica de la economía tiene su primer gran exponente en el libro "Economía para entender" publicado inicialmente en 1995 y reeditado y revisado tres años después bajo el título de "Guía para entender de economía" Pulido (1998:1), y donde, en palabras del propio autor "se pueden encontrar respuestas a muchas de esas preguntas que siempre se ha hecho. 
¿Qué piensan y saben los políticos de economía? ¿Por qué los economistas tienen opiniones tan diferentes? ¿Qué hace que suba o baje la peseta en relación al marco o al dólar? ¿Tiene solución el problema del paro? ¿Por qué cuesta tanto el Estado?... "No sólo es un libro que intenta divulgar los conocimientos económicos más actuales. Su visión es lo suficientemente amplia como para tocar cuestiones ideológicas, de ética o de geopolítica. Su estilo es tan directo como para que el autor nos haga participes de sus preocupaciones, de sus críticas a las medidas adoptadas durante los últimos años y de sus propias propuestas de actuación." Pulido (1995:6). Este libro fue revisado y reeditado tres años más tarde bajo el título de "Guía para entender de economía" Pulido (1998:1)

Esta labor de divulgación tuvo su continuidad en la colección "Conocer las claves de la economía", publicada en 1996, y en la que se abordan temas como la política económica de gobiernos y empresas Pulido (1996:3); el comercio internacional, Pulido (1996:4); política y economistas, Pulido (1996:5); los datos económicos, Pulido (1996:6); o empleo, inflación y sector público, Pulido (1996:7).

Un año más tarde, Antonio Pulido, dirigió la colección Economía XXI editada por Pirámide y donde el autor revisa diferentes temas de actualidad como la entrada en la Comunidad Europea Pulido (1997:2), los efectos de la incipiente globalización, Pulido (1997:3); las posibilidades de la economía española en el contexto internacional, Pulido (1997:4), conceptos básicos de economía financiera y distintas teorías económicas alternativas, Pulido (1997:5), prospectiva económica mundial Pulido (1998:2); o el proceso de creación y entrada en la moneda única europea, Pulido (1998:3).

Este afán por la divulgación económica, llevó al autor a crear en su obra "Viajes por Econolandia" Pulido (2002:4) un país imaginario los Estados Unidos de Econolandia con su capital Ciberville, y una serie de personajes arquetípicos como el periodista económico Larry Newsletter, co-autor del libro, el profesor de economía Juan Macro, el especialista en "nueva economía" Taco Novo, el consultor internacional Peter World, el empresario John Business, o el futurólogo Máximo Riesgo, y que le sirven para introducir de forma novelada, y con la estructura de un libro de viajes, todos los grandes conceptos macroeconómicos y presentar algunos de los problemas derivados de la globalización y la nueva economía.

Este mismo país imaginario, Econolandia, y su co-autor ficticio Larry Newsletter, sirven de marco para la segunda entrega de divulgación económica novelada que publicó el autor en el año 2006 bajo el título de "Momentos estelares de Econolandia", Pulido (2006:4), y donde un viaje en el tiempo nos lleva a lugares como Córdoba y Constantinopla en 970; Toledo en 1270; Venecia en 1470; Medina del Campo, Salamanca y Sevilla en 1570; París y Londres en 1670; Madrid, París y Glasgow en 1770; Londres en 1870; Nueva 
York y Cambridge en 1929; Mil Islas, Canadá, en 2013, para ilustrar diversos fenómenos económicos y sociales mediante conversaciones directas con personajes reales como Fra Luca Pacioli en el siglo XV; Simón Ruiz, Teresa de Jesús, Fray Luis de León o Tomás de Mercado en el siglo XVI; William Petty o Jean-Baptiste Colbert en el XVII; el Conde de Campomanes, François Quesnay o Adam Smith en el XVIII; Karl Marx en el XIX y con Keynes ya en el siglo XX.

Toda esta labor de divulgación económica ha tenido su continuidad a través de una web creada por el propio autor (www.econolandia.es) donde nos encontramos con un diccionario económico que recoge definiciones accesibles de una buen grupo de conceptos económicos, el rincón del profesor, con iniciativas pedagógicas en economía, las principales estadísticas y datos macroeconómicos, cursos de economía, todos tipo de documentos técnicos y de divulgación, e incluso un trivial para contrastar nuestros conocimientos de economía.

\section{OTROS TEMAS}

Finalizaremos esta revisión de la obra de Antonio Pulido incorporando otras contribuciones que ha ido realizando a lo largo de su dilatada carrera y que, estando vinculadas con todos los aspectos recogidos en los apartados precedentes, no tenían un encaje directo en ninguno de ellos en concreto.

Así, por ejemplo, en un documento de trabajo de 1992, realiza una reflexión general sobre su propia labor a lo largo de 25 años de trabajo en la economía aplicada. Pulido (1992:4).

De la misma forma y, como es bastante habitual en la práctica científica, ha participado en diversas obras de reconocimiento de otros colegas de profesión, tales como Gerard Debreu, Pulido (1984:6); José Barea, Pulido (2011); a su gran amigo y colega, Emilio Fontela, Pulido (2007:8); y, por supuesto, al gran maestro de la econometría aplicada el Profesor Lawrence R. Klein, Pulido (1980:4, 1980:5, 1981:6, 1981:7, 2014, 2015:2) y Pulido y Pérez (2006:3).

Aun cuando, la mayor parte de su carrera profesional se ha centrado en la economía aplicada, en general, y en la econometría, en particular, sus primeros años como docente los dedicó, parcialmente al marketing y la distribución comercial, siendo director de la revista Cuadernos Universitarios de Planificación Empresarial y Marketing entre 1975 y 1982, y habiendo realizado varias publicaciones en esta área, Pulido (1966:6, 1966:7, 1967:3, 1967:4, 1968:3, 1969:4, 1969:5, 1969:6, 1970:1, 1970:2, 1974:2, 1974:3, 1974:4, 1974:5, 1974:6, 1975:2, 1976:3, 1976:4, 1976:5, 1980:6), Montgomery y otros (1976).

En su calidad de económetra aplicado, siempre ha mostrado un interés especial en los procesos de recopilación y tratamiento estadístico habiendo sido 
Consejero del Instituto de Estadística de Castilla-La Mancha, miembro del Consejo Asesor de "Fuentes Estadísticas" (1994-2004), miembro del Consejo Superior de Estadística (1990-1997) y presidente de la Ponencia del Plan Estadístico Nacional (1991-1996); y publicando diversos trabajos vinculados, precisamente, con esta labor estadística, Pulido (1981:8, 1987:3, 1994:4, 1994:5, 2003:1), Fontela y Pulido (1991).

Por último, aunque no por ello menos relevante, el desarrollo sostenible y el medio ambiente han sido otros de los temas objeto de atención por parte de este autor a lo largo de su trayectoria investigadora, habiendo publicado diversos artículos y documentos de trabajo, Pulido (1982:7, 2003:2, 2004:4, 2008:6, 2008:7), Pulido y otros (1980:2, 2001:2) y siendo co-autor, junto con Emilio Fontela del libro "Principios de desarrollo sostenible" y donde ambos autores se plantean la economía como una ciencia social, condicionada por múltiples aspectos no económicos (políticos, tecnológicos, psicológicos,...) y que debe estar a disposición de la humanidad para conseguir sus metas de bienestar material y calidad de vida. Pulido y Fontela (2004).

\section{BREVE RESEÑA BIOGRÁFICA}

Antonio Pulido, nació en Febrero de 1940 y se obtuvo la diplomatura de estadística y la licenciatura de economía en 1963 en la Universidad Complutense de Madrid, donde se doctoró dos años después en Ciencias Económicas y Comerciales, siendo su director de tesis Ángel Alcaide.

$\mathrm{Su}$ trayectoria docente se inició en el curso 1961/1962, como profesor ayudante en la Universidad Complutense de Madrid y adquirió la adjuntía en esa misma universidad en el curso 1964/1965. En 1971, accedió a una cátedra en la Universidad de Valencia, donde se mantuvo durante dos cursos académicos, hasta que obtuvo una nueva cátedra en el Universidad Autónoma de Madrid de la que tomó posesión en el año 1973 y donde actualmente continúa su actividad como Catedrático Emérito desde el año 2010.

Aunque su carrera profesional es fundamentalmente docente e investigadora, no ha permanecido, ni mucho menos, ajeno al mundo empresarial y de la administración pública, con el que ha mantenido vinculaciones permanentes a través del Instituto "L.R.Klein" de la Universidad Autónoma de Madrid y de la Asociación Centro de Predicción Económica CEPREDE, y en el que estuvo directamente involucrado en los inicios de su trayectoria, como asesor del departamento de estudios de Alas publicidad entre 1962 y 1963, y también como asesor de la Secretaría General Técnica del Ministerio de Trabajo de 1964 a 1965, como técnico y posteriormente subdirector de estudios empresa consultora Sociedad de Investigación Económica, entre 1964 y 1967, y también en ese mismo periodo actuó como director del Gabinete de estudios económicos 
del Instituto de Estudios Turísticos. En 1968 fue Secretario General de Cointra y en 1969 fundó la consultora Macrométrica que presidió hasta el año 1975.

En su actividad docente ha ocupado diferentes cargos de gestión y ha participado en diversas comisiones y grupos de trabajo. Entre 1973 y 1978 fue Director del Departamento de Investigación Comercial; y en el período 19791985, Director del Departamento de Econometría de la Empresa e Informática; posteriormente en los cursos 1986/1987 y 1990/1991, dirigió el Departamento de Economía Aplicada de la Facultad de Ciencias Económicas y Empresariales de la Universidad Autónoma de Madrid. Desde 1987 hasta el año 2003 fue miembro del Consejo Directivo y Profesor del Master Business Administration del Instituto Universitario de Administración de Empresas (IADE) de la Universidad Autónoma de Madrid, y durante los últimos años es miembro del Grupo de Trabajo de profesores eméritos para la elaboración del Plan Estratégico de la Facultad de Ciencias Económicas y Empresariales de la Universidad Autónoma de Madrid.

Al margen de los cargos estrictamente académicos, ha sido miembro de la Comisión asesora del programa ACADEMIA de la Agencia Nacional de Evaluación de la Calidad y Acreditación (ANECA), Presidente del Comité de Ciencias Sociales y Jurídicas del Programa de Evaluación del Profesorado de la Agencia Nacional de Evaluación de la Calidad y Acreditación (ANECA) y Coordinador del subcomité de Ciencias Sociales y Jurídicas.(2006-2007); Consejero del Instituto de Estadística de Castilla-La Mancha, miembro del Consejo Superior de Estadística (1990-1997) y Presidente de la Ponencia del Plan Estadístico Nacional (1991-1996); miembro de la Comisión de Seguimiento de España.es creada por la D.G. de la Sociedad de la Información del Ministerio de Ciencia y Tecnología y del Consejo Económico y Social de la Comunidad de Madrid). (1996-2000).

En el ámbito de la difusión, es miembro de diversos consejos editoriales de revistas científicas y ha dirigido tanto la revista Cuadernos Universitarios de Planificación Empresarial y Marketing entre 1975 y 1982 y de la Revista de Estudios de Economía Aplicada entre los años 2003 y 2008.

A lo largo de su trayectoria profesional ha recibido diverso premios $\mathrm{y}$ reconocimientos entre los que destaca el Premio de Economía de Castilla y León "Infanta Cristina" que recibió en el año 2005.

\section{REFERENCIAS BIBLIOGRÁFICAS}

ALCAIDE, A. (1957). "Estudio de un modelo econométrico del mercado de calzado en España desde 1940 a 1955". I Centenario de la Estadística Española, I.N.E. 
ANTILLE, G, E. FONTELA, y A. PULIDO (2005). "Input-output in Europe: Trends in research and applications" $15^{\text {th }}$ International Input-output Conference. Beijing, China P.R. 2005

CASTAÑEDA, J. (1945). "El consumo de Tabaco en España y sus factores". Instituto de Estudios Políticos. Madrid.

DONES, M. y otros (2005). "Modelo de simulación de la economía madrileña para la proyección de variables demográficas y macroeconómicas". En Fontela (Ed.): La Comunidad de Madrid: Prospectiva 2015. pp. 135-161.

DONES, M., J. PÉREZ y C. LLANO (2009). "An interregional impact analysis of the EU Structural Funds in Spain (1995-1999)". Papers in Regional Science. Vol.88(3), pp. 509-530.

DONES, M.; J. PÉREZ y A. PULIDO (2008). "El impacto macroeconómico de la biotecnología". Medicina Clínica, volumen 131-Extraordinario 2, Noviembre 2008. pp. 60-65.

DONES, M., J. PÉREZ y A. PULIDO (2010). “L'impacte econòmic de la biotecnologia a Catalunya". Nota d'Economia, Nº. 97-98, pp. 43-65.

DONES, M. y A. PULIDO (1986). "Estrategias de políticas económicas: comparación internacional". Instituto Lawrence R. Klein, Documento 86/ME-4.

ECHENIQUE, M. (1956). "Análisis estadístico de la demanda de azúcar en España". Revista de Economía Política, № 15, pp. 100-170.

FONTELA, E. y A. PULIDO (1973). "Análisis de las repercusiones sectoriales de variaciones en demanda y precios". Dirección General de Promoción y Tecnología.

FONTELA, E. y A. PULIDO (1986). "Input-output, technical change and the long waves". Documento Instituto “L.R.Klein" 86/6 1.

FONTELA, E. y A. PULIDO (1989:1). "Input-Output and technological change". Documento $D S T I / S P R / 89.7$. Seminaire International sur la Science, la Technologie et la Croissance economique, OCDE.

FONTELA, E. y A. PULIDO (1989:2). "Progrès technologique et input-output". En Artis y Suriñach (Eds): Jornadas sobre modelización económica. Universidad de Barcelona. págs. 235-250.

FONTELA, E. y A. PULIDO (1990:1). "Las tablas input-output y su integración en el sistema de cuentas nacionales". Instituto Lawrence R. Klein, Documento 90/2.

FONTELA, E. y A. PULIDO (1990:2). "Input-output et surplus de productivité: la economie espagnole entre 1975 et 1980". En Archambault Arkhipoff, (eds): Etudes de Comptabilité Nationale. París: Económica.

FONTELA, E. y A. PULIDO (1991). "Comptabilité nationale et prévision macroéconomique".Instituto Lawrence R. Klein, Documento 91/5.

FONTELA, E. y A. PULIDO (1992). "Predicción y simulación en modelos Input-Output". Instituto Lawrence R. Klein, Documento 92/8.

FONTELA, E. y A. PULIDO (2003). "Efectos sobre la economía española Seis variantes para estudiar el cambio estructural de la UE". Índice revista de estadística y sociedad, No. 1, pp. 8-9.

FONTELA, E. y A. PULIDO (2005). "Tendencias de la investigación en el análisis Inputoutput". Revista Asturiana de Economía, № 33 2005, pp. 9-29.

FONTELA, E. y otros (1989). "University enterprise relations in OECD Member Countries". Documento DSTI/SPR/89.37, OCDE. 
FONTELA, E. y otros (1990). "Committee for scientific and technological policy universityenterprise relations in OECD member countries". Documento DSTI/SPR/89.37, OCDE 1989 Organisation for Economic Coopertation and Development.

FONTELA, E. y otros (1992). "Evaluación de la actuación del CDTI en apoyo a la I + D". Centro para el Desarrollo Tecnológico Industrial.

GARCÍA BARBANCHO, A. (1961). "Aplicación del Modelo de Harrod-Domar a la economía española". Estadística Española, 12, pp. 26-35.

LLANO, C. y otros (2001). "Una visión de conjunto del comercio de la Comunidad de Madrid con el resto de España y con el extranjero". En Mella y Sanz, (eds): Balanza de Pagos de la Comunidad de Madrid (1995-1998)", pp. 143-162.

LLANO, C. y otros (2009). "La base de datos C-intereg sobre el comercio interregional de bienes en España: método y primeros resultados (1995-2006)". EKONOMIAZ. n: 69, pp. $244-269$.

LLANO, C. y otros (2010). "Opening the Interregional Trade Black Box: The C-intereg Database for the Spanish Economy (1995-2005)". International Regional Scientific Review, vol.: 33, no:3, pp. $302-337$.

LLANO, C. y A. PULIDO (2002). "Estructura espacial y sectorial del comercio de Andalucía y su efecto sobre el crecimiento". Boletín Económico de Andalucía 2002 Boletín económico de Andalucía, № 31-32, 2002, pp. 47-62.

LÓPEZ, A.M. y A. PULIDO (2000). "Aproximación al análisis de las cadenas de producción sectoriales para España y Asturias a partir de las tablas input-output". Instituto Lawrence R. Klein, UAM.

LÓPEZ, A.M. y A. PULIDO (2002). "Modelización de la difusión regional de las Nuevas Tecnologías". Cuadernos del Fondo de Investigación Richard Stone, № 4, Instituto L.R. Klein - Centro Stone.

LÓPEZ, A.M. y A. PULIDO (2003:1). "Madrid: economía dinámica". Economistas, № 95, (Ejemplar dedicado a: Economía de Madrid), pp. 21-27.

LÓPEZ, A.M. y A. PULIDO (2003:2). "La Nueva Economía en Andalucía: comparativa regional". Boletín económico de Andalucía, № 33-34, (Ejemplar dedicado a: Nueva Economía y Sociedad del Conocimiento), pp. 77-90.

LÓPEZ, A.M. y A. PULIDO (2003:3). "Modelización econométrica de la difusión regional de las nuevas tecnologías". 27 Congreso Nacional de Estadística e Investigación Operativa. Lleida, 2003.

LÓPEZ, A.M. y A. PULIDO (2005). "Ahorro y consumo como factores de crecimiento". En: "Crecimiento y competitividad: bases del progreso económico y social", Federación de Cajas de Ahorros Vasco-Navarras, pp. 93-113.

LÓPEZ, A.M. y A. PULIDO (2010). "Perspectivas de crecimiento económico, ¿cuándo saldremos de la crisis?". Investigación y marketing, №. 106, pp. 14-21.

MARTÍN BLANCO, M. (1962). "Análisis econométrico del Sector eléctrico". Revista de Ciencias Aplicadas. Julio - Agosto.

MARTÍNEZ AGUADO, T. (1979). "Base de datos del modelo Wharton-UAM. Series homogéneas 1954-1978". Proyecto de predicción económica, Documento 79/5, Universidad Autónoma de Madrid.

MONTGOMERY, D.B. y otros (1976). Marketing científico. Madrid: Ediciones Pirámide.

MUR, A. y A. PULIDO (1992). "Contraste de un modelo de predicción input- output". Estudios de economía aplicada: VI Reunión Anual de ASEPELT-España, 4 y 5 de junio de 1992, Vol. 2, pp. 401-410. 
PENA, J.B. (1960). "Modelo econométrico para el cálculo y predicción de una población total, activa y migrante a escala regional". Estadística Española, 9.

PÉREZ, J., C. LLANO y G. GARCÍA (2009). "Valoración de las Tablas Input-Output Interregionales de la economía española". Monográfico ICE. Aspectos territoriales del desarrollo cara al siglo XXI. Vol. 88, pp. 67-88.

PÉREZ, J., y A. PULIDO (2001). "Hacia una valoración del impacto macroeconómico de las TIC". XV Reunión de Aspelt 2001.

PÉREZ, J., y A. PULIDO (2005). "Propuesta metodológica para la evaluación de la calidad docente e investigadora: planteamiento y experimentación". Anales de economía aplicada 2005, pp. 211.

PULIDO, A. (1965). "Las estadísticas de ventas de gasolina y su aprovechamiento a fines del análisis del Turismo". Estudios Turísticos.

PULIDO, A. (1966:1). "Introducción a un análisis econométrico del turismo". Instituto de estudios Turísticos. Cuadernos Monográficos $n^{\circ} 5$.

PULIDO, A. (1966:2). "Especificación de modelos econométricos de desarrollo". Economía Política, № 43-44, 1966, pp. 35.

PULIDO, A. (1966:3). "Análisis econométrico del nivel de empleo". Revista de Trabajo,

PULIDO, A. (1966:4). "Técnicas econométricas de planificación de la educación: el modelo de Tinbergen". Anales de Economía, №. 14, pp. 181-198.

PULIDO, A. (1966:5). "Aplicación de la econometría al análisis del fenómeno turístico". Estudios Turísticos.

PULIDO, A. (1966:6). "Investigación Operativa y Publicidad". Boletín de Estudios Económicos, Vol. 22, № 68 (MAY-AGO), pp. 445-510.

PULIDO, A. (1966:6). "Estudio sobre medida de la publicidad en España". Control de Publicidad y Ventas.

PULIDO, A. (1967:1). "Estadística y técnicas de investigación social". Ed. Gráficas Caduceo. Ediciones posteriores de Anaya y Pirámide.

PULIDO, A. (1967:2). "Aplicación de la econometría al análisis del turismo: modelos econométricos". En "Problemas socioeconómicos y técnicos del desarrollo turístico", Instituto de Estudios Turísticos.

PULIDO, A. (1967:3). "Estudios de penetración de campañas publicitarias". Primeras Jornadas de Marketing, 1967.

PULIDO, A. (1967:4). "Distribución óptima de un presupuesto de publicidad". Control de Publicidad y Ventas.

PULIDO, A. (1968:1). "La función de producción Cobb-Douglas". Riqueza Nacional de España, Deusto.

PULIDO, A. (1968:2). "Balanza de Pagos y Contabilidad Nacional del fenómeno turístico". Estudios turísticos, №. 19, pp. 5-120.

PULIDO, A. (1968:3). "Investigación Comercial, Medición y programación de la publicidad". ESIC.

PULIDO, A. (1969:1). "Posibilidades de aplicación del proceso electrónico de datos a la elaboración de modelos econométricos de política general". Centro de Cálculo de la Universidad de Madrid.

PULIDO, A. (1969:2). "Perspectivas de la econometría". Información Comercial Española, ICE: Revista de economía, № 435, 1969, pp. 53-58. 
PULIDO, A. (1969:3). "Métodos econométricos de política laboral en España". Información Comercial Española, № 435, 1969, pp. 69-76.

PULIDO, A. (1969:4). "Evolución y revolución del Marketing”. Marketing Actualidad.

PULIDO, A. (1969:5). "Marketing, Investigación Comercial e Investigación de Mercados". Control de Publicidad y Ventas.

PULIDO, A. (1969:6). "La planificación en el desarrollo y lanzamiento de nuevos productos". Terceras Jornadas de Marketing 1969.

PULIDO, A. (1970:1). "Planteamiento del problema de medición y programación de la publicidad". ESIC-Market .

PULIDO, A. (1970:2). "La previsión de venta y su aplicación a la planificación del marketing". XI Seminario AEDEMO 1975.

PULIDO, A. (1971:1). "Estimación de modelos econométricos: principios básicos del método de la máxima verosimilidad". En "Lecturas sobre Econometría", Ed. Gredos.

PULIDO, A. (1971:2). "Tratamiento econométrico de series temporales aisladas. I.Descomposición temporal”. Documento de trabajo Universidad de Valencia.

PULIDO, A. (1971:3). "El modelo español de desarrollo educativo y el análisis costeeficacia". Hacienda Pública.

PULIDO, A. (1972). "Utilización de modelos econométricos para la valoración de proyectos de transporte". Revista Española de Economía, Vol. 2, № 3 (SEP-DIC), 1972, pp. 167-193.

PULIDO, A. (1973:1). "Una aproximación econométrica a la problemática del desarrollo regional". Boletín de estudios económicos, Vol. 28, № 89 (AGO), pp. 553-586.

PULIDO, A. (1973:2). "Análisis de los factores de crecimiento económico". Boletín del Fondo para la Investigación Económica y Social, Vol. 5, №. 3 (JUL-SEP), pp. 392403.

PULIDO, A. (1974:1). Tratamiento Econométrico de la Inversión. Madrid: Ed. Aguilar.

PULIDO, A. (1974:2). “Evolución y Revolución del Marketing”. Editorial: Asociación para el Progreso de la Dirección.

PULIDO, A. (1974:3). "La planificación del marketing, previsiones, control y análisis de resultados". Jano Management.

PULIDO, A. (1974:4). "Las nuevas formas comerciales en los distintos niveles de desarrollo". En "Nuevas formas comerciales", IRESCO.

PULIDO, A. (1974:5). "Presente y futuro del marketing en España”. Dirección y Progreso.

PULIDO, A. (1974:6). "Informe sobre la enseñanza del marketing". Marketing Actualidad.

PULIDO, A. (1974:7). "Hacia un marketing científico". Marketing Actualidad.

PULIDO, A. (1975:1). "Métodos y modelos econométricos". En "Informática y Planificación", Centro de Cálculo de la Universidad Complutense 1975.

PULIDO, A. (1975:2). "Modelos de demanda de nuevos productos". Cuadernos Universitarios de Planificación Empresarial, Vol. 1 n¹, pp. 63-119.

PULIDO, A. (1976:1). "Hacia una teoría de los cambios en las formas comerciales". Boletín de Estudios Económicos, Vol. 31, № 97 (ABR), pp. 9-31.

PULIDO, A. (1976:2). "Las tablas input-output como herramienta de análisis de marketing y turismo". Estudios turísticos, №. 49-50, (Ejemplar dedicado a: Las Tablas inputouput), pp. 133-139. 
PULIDO, A. (1976:3). "Determinación del presupuesto de publicidad". Cuadernos Universitarios de Planificación Empresarial y Marketing, Vol. 2, n² 2, pp.171-199.

PULIDO, A. (1976:4). "Características e implicaciones de los hipermercados". Información Comercial Española, № 510, pp. 71-82.

PULIDO, A. (1976:5). "Macro y micro distribución". Cuadernos Universitarios de Planificación Empresarial y Marketing, Vol. 2, №. 4, pp. 347-352.

PULIDO, A. (1977:1). "Modelos econométricos del cambio tecnológico". Libre Empresa.

PULIDO, A. (1977:2). "Los ciclos económicos". Universidad Nacional de Educación a Distancia.

PULIDO, A. (1978:1). "Los modelos econométricos como base para la adopción de decisiones empresariales". Cuadernos Universitarios de Planificación Empresarial y Marketing, Vol. 4, №. 3, pp. 317-337.

PULIDO, A. (1978:2). "La evolución del desempleo en España. Un ejercicio de predicción a corto plazo". Cuadernos Universitarios de Planificación Empresarial y Marketing, Vol. 4, No. 3, pp. 243-268.

PULIDO, A. (1979:1). "La cuantificación de los movimientos turísticos". En "Turismo: Horizonte 85", Editur.

PULIDO, A. (1979:2). El reto de la investigación para la empresa. Editorial: Forum Universidad Empresa.

PULIDO, A. (1980:1). "Hacia un enfoque econométrico de la planificación estratégica de la empresa". En Estudios monográficos de contabilidad y de economía de la empresa: en homenaje al profesor Doctor José María Fernández Pirla. pp. 609-630.

PULIDO, A. (1980:2). "Perspectivas regionales en la década de los 80". En Regional Development in Spain. UNRISD/Monton.

PULIDO, A. (1980:3). "Perspectivas económicas y posibilidades de la economía española en los inicios de los 80". Revista de Comercio Exterior, Banco Nacional de Comercio Exterior, Vol. 30, № 6, México.

PULIDO, A. (1980:4). "Elogio de Lawrence Klein, doctor "honoris causa" por la Universidad Autónoma de Madrid". Cuadernos Universitarios de Planificación Empresarial, Vol. 6, №. 4, pp. 639-646.

PULIDO, A. (1980:5). "Carta abierta a Lawrence Klein, nuevo Premio Nobel de Economía ". El País.

PULIDO, A. (1980:6). "Predicción en Marketing: del ordenador a la bola de cristal". Congreso de Investigación y Marketing AEDEMO 1980.

PULIDO, A. (1981:1). "Simulación con modelos econométricos. La experiencia WhartonUAM de elaboración de un modelo para la economía española". Universidad de Deusto.

PULIDO, A. (1981:2). "Posibilidades y limitaciones de la investigación en la Universidad y su relación con la empresa“. En: La Universidad española actual. Universidad de Zaragoza.

PULIDO, A. (1981:3). "La contribución de la empresa privada al desarrollo económico y social y perspectivas en la década de los 80". CEOE, Informes y Estudios.

PULIDO, A. (1981:4). "Algunos datos sobre el problema del empleo". Fundación Universidad Empresa. Coloquio sobre el empleo de los Universitarios 1981.

PULIDO, A. (1981:5). "Escenarios para una crisis". Cuadernos Universitarios de Planificación Empresarial, Vol. 7, №. 2, pp. 297-305. 
PULIDO, A. (1981:6). "Una presentación para no especialistas del Premio Nobel de Economía de 1980, Lawrence Klein y sus modelos econométricos". Investigación y Ciencia, № 52 , pp. 42-43.

PULIDO, A. (1981:7). "Apuntes sobre la obra de Lawrence R. Klein". Revista española de economía, № 1, 1981 (Ejemplar dedicado a: Homenaje a Lawrence R. Klein, Premio Nobel de Economía 1980), pp. 17-36.

PULIDO, A. (1981:8). "Estadísticas de Rentas y Precios". Jornadas de Estadística Española, INE 1981.

PULIDO, A. (1982:1). "Introducción a las técnicas de predicción empresarial. I.- Modelos econométricos". Escuela de Economía.

PULIDO, A. (1982:2). "El proyecto LINK y la predicción económica mundial". Terceras Jornadas de Perspectivas Económicas y Empresariales.1982.

PULIDO, A. (1982:3). "Planificación, evaluación y seguimiento de la investigación universitaria". Seminario sobre "La gestión de la investigación en las Universidades", Fundación Universidad-Empresa.1982.

PULIDO, A. (1982:4). "Establecimiento de prioridades de investigación y actuación en el terreno de las ciencias ambientales. Metodología general previa". CEOIMA-CIFCA.

PULIDO, A. (1982:5). "Investigación económica y medio ambiente". Seminario sobre "Desarrollo, economía y medio ambiente", CIFCA.1982.

PULIDO, A. (1982:6). "Algunos impactos de las tecnologías y los medios de información sobre la economía en su conjunto, con especial referencia a los aspectos de gestión, productividad y empleo". Fundesco.

PULIDO, A. (1982:7). "Investigación económica y medio ambiente". Seminario sobre "Desarrollo, economía y medio ambiente", CIFCA, 1982.

PULIDO, A. (1983:1). Modelos econométricos. Madrid: Ed. Pirámide.

PULIDO, A. (1983:2). "Los modelos econométricos: una herramienta útil para la planificación". Dirección y Progreso, $\mathrm{n}^{\circ} 71$.

PULIDO, A. (1983:3). "Proceso de elaboración y rectificación de predicciones". Documentos CEPREDE, N5, pp. 36-39.

PULIDO, A. (1983:4). "Rentas, ahorro y necesidades de financiación". Documentos CEPREDE, No5, pp. 1-25.

PULIDO, A. (1983:5). "Inversión y sus componentes", Documentos CEPREDE. №5, pp. 26-36.

PULIDO, A. (1983:6). "Medición de los efectos económicos de la investigación". Fundación Empresa Pública, OGEINAP.

PULIDO, A. (1983:7). "Predicción y simulación de los efectos económicos de y sobre la investigación en España". Fundación Empresa Pública, OGEINAP.

PULIDO, A. (1983:8). "El economista ante el reto del futuro". Boletín del Colegio de Economistas de Madrid.

PULIDO, A. (1983:9). "Escenario económico español en los próximos años". En Profesiones con futuro. Achna.

PULIDO, A. (1984:1). "Las previsiones de inflación y de tipos de interés como base de la planificación de la inversión en activos financieros". En Inversión en Activos Financieros. Instituto de Empresa.

PULIDO, A. (1984:2). "Análisis de errores de predicción en la experiencia Ceprede 198183". Documentos CEPREDE. № 7. 
PULIDO, A. (1984:3). " Previsión de la economía española a escala regional". Información Comercial Española, ICE: Revista de economía, № 609, pp. 121-128.

PULIDO, A. (1984:4). "Estudiantes universitarios: orientación, información y futuro". Ministerio de Educación y Ciencia.

PULIDO, A. (1984:5). "El futuro de los estudios universitarios". Fundación UniversidadEmpresa.

PULIDO, A. (1984:6). "Gerard Debreu, Nobel de Economía 1983". Investigación y Ciencia.

PULIDO, A. (1986:1). "Modelo Wharton-UAM de la economía española: su estructural causal básica". En Libro homenaje a Gonzalo Arnaiz. Instituto Nacional de Estadística.

PULIDO, A. (1986:2). "Modelos macroeconométricos como soporte para predicciones sectoriales". En: Predicción y economía de las telecomunicaciones. Fundesco.

PULIDO, A. (1986:3). "Crecimiento económico y esfuerzo tecnológico. Un enfoque integrado, modelo econométrico/modelo input-output con coeficientes variables". Instituto Lawrence R. Klein, Documento 86/1.

PULIDO, A. (1986:4). "Las nuevas profesiones y los sectores con futuro". En: Enseñanza universitaria y mercado de trabajo: el primer empleo de los titulados universitarios, pp. 171-181.

PULIDO, A. (1987:1). "Servicio de seguimiento y predicción a corto plazo: indicadores de demanda". Documentos CEPREDE. 87/3.

PULIDO, A. (1987:2). "Algunas iniciativas pedagógicas en la enseñanza de la economía". Documento IADE 87/5, U.A.M 1987

PULIDO, A. (1987:3). "Contabilidad Nacional y Modelos Econométricos". Instituto Lawrence R. Klein, Documento 87/3.

PULIDO, A. (1988:1). "Experiencias con modelos macroeconométricos: luces y sombras keynesianas". En Rafael Rubio de Urquía (aut.): La herencia de Keynes. 479 págs.

PULIDO, A. (1988:2). "Técnicas elementales de predicción". Instituto Lawrence R. Klein, Documento 88/7.

PULIDO, A. (1988:3). "Técnicas avanzadas de predicción”. Instituto Lawrence R. Klein, Documento 88/8.

PULIDO, A. (1988:4). "El enfoque de la predicción”. Instituto Lawrence R. Klein, Documento 88/5.

PULIDO, A. (1988:5). “Técnicas de análisis regional”.En Mella (ed.): Economía y política regional en España ante la Europa del siglo XXI. Asociación española de ciencia regional, pp. 96-109.

PULIDO, A. (1989:1). Predicción Económica y Empresarial. Madrid: Ed. Pirámide.

PULIDO, A. (1989:2). "Productividad, inflación y retribución de factores: aplicación del MAP a la economía española". En Artís y Suriñach (eds): Modelización Económica. Barcelona: Universidad de Barcelona. pp. 251-284.

PULIDO, A. (1989:3). "Modelización de tipos de interés". Estudios de Economía Aplicada, Segunda Reunión Anual ASEPELT-ESPAÑA. Universidad de Valladolid. Serie Economía № 10, pp. 165-199.

PULIDO, A. (1990:1). "La enseñanza de economía aplicada asistida por ordenador". Instituto Lawrence R. Klein, Documento 90/4 1

PULIDO, A. (1990:2). "El futuro económico de las regiones españolas. El futuro proyecto HISPALINK". Instituto Lawrence R. Klein, Documento 90/5. 
PULIDO, A. (1990:3). "Hacia una clasificación de las Universidades según criterios de calidad, (introducción)". Forum Universidad-Empresa.

PULIDO, A. (1991:1). "Primeras experiencias con el modelo de congruencia HISPALINK". Instituto Lawrence R. Klein, Documento 91/2.

PULIDO, A. (1991:2). "España, ¿modelo de crecimiento para México?”. Instituto Lawrence R. Klein, Documento 91/11.

PULIDO, A. (1991:3). "Estrategia empresarial en entornos cambiantes". Instituto Lawrence R. Klein, Documento 91/1.

PULIDO, A. (1992:1). "Técnicas de predicción a nivel profesional". En Ponencias III Reunión Asepelt. Biblioteca de Socioeconomía Sevillana, Diputación de Sevilla 1992.

PULIDO, A. (1992:2). "Propuesta Metodológica para el diseño de un modelo de análisis regional integrado con desagregación sectorial". Estudios de Economía Aplicada: VI Reunión Anual de ASEPELT-España, 4 y 5 de junio de 1992, Vol. 2, 1992, pp. 341356.

PULIDO, A. (1992:3). "Dinámica de crecimiento de las regiones españolas: una visión integrada según el Proyecto Hispalink". En Pulido y Cabrer (eds.): Datos, técnicas y resultados del moderno análisis económico regional: Proyecto Hispalink. Jornadas Hispalink (8 . 1992. Valencia), pp. 399-431.

PULIDO, A. (1992:4). "25 años de experiencia en econometría aplicada". Instituto Lawrence R. Klein, Documento 92/1"

PULIDO, A. (1993). "25 años de experiencia en econometría aplicada". Estudios de Economía Aplicada, № 0, 1993, pp. 81-98.

PULIDO, A. (1994:1). "Condicionantes nacionales e internacionales de la economía andaluza". En VV.AA.: La economía andaluza ante la Unión Europea. Caja San Fernando de Sevilla y Jerez.

PULIDO, A. (1994:2). "Globalización empresarial: Nuevas metas, nuevas estrategias". Instituto Lawrence R. Klein, Documento 94/6.

PULIDO, A. (1994:3). "La competitividad de la empresa española". Serie Grandes Temas, N ${ }^{\circ} 7$. Instituto de la Comunicación Pública, S.A.

PULIDO, A. (1994:4). "¿Por qué debe usted conocer el Plan Estadístico Nacional?". Fuentes Estadísticas (INE), № 1, pp. 3-4.

PULIDO, A. (1994:5). "Evaluación y perfeccionamiento de la estadística española". Economistas Año № 12, № 59, pp. 12-15.

PULIDO, A. (1995:1). "Integración económica regional". En Cabrer, B. (ed.): La integración económica regional en España: La comunidad valenciana. Proyecto Hispalink pp. 1726.

PULIDO, A. (1995:2). "Panorámica de la modelización econométrica regional". Cuadernos Aragoneses de Economía. 2a época, volumen 4, № 21995 pp. 211-229.

PULIDO, A. (1995:3). "Reflexiones sobre el análisis input-output del comercio interregional". Documento de trabajo Instituto "L.R.Klein". UAM.

PULIDO, A. (1995:4). "Desequilibrios de la economía española". Investigación y Ciencia $1995 \mathrm{~N}^{\circ} 231$, pp. 6-13.

PULIDO, A. (1995:5). “¿Tiene sentido recortar la inversión pública?”. Seopan.

PULIDO, A. (1995:6). Economía para entender. Madrid: Editorial Pirámide.

PULIDO, A. (1996:1). "Input-output regional: posibilidades y limitaciones". En El Desarrollo de las regiones, nuevos escenarios y perspectivas de análisis: XXII. Reunión de 
Estudios Regionales. Pamplona-Iruña, 20-22 de noviembre de 1996, Vol. 1, 1996 (Ponencias), pp. 265-286.

PULIDO, A. (1996:2). "Déficit e inversión pública: la conexión oculta“. En Inversión pública $y$ déficit. Ediciones Gestión 2000, S.A.

PULIDO, A. (1996:3). "Política económica de gobiernos y empresas: ¿actuar o sufrir?". Colección: Conocer las claves de la economía. Madrid: Editorial Negocios/Pirámide.

PULIDO, A. (1996:4): “Comerciar con el mundo: ¿guerra o colaboración?”. Colección: Conocer las claves de la economía. Editorial: Negocios/Pirámide.

PULIDO, A. (1996:5): "Economía y Políticas - Política y Economistas. Colección: Conocer las claves de la economía". Colección: Conocer las claves de la economía" Editorial: Negocios/Pirámide.

PULIDO, A. (1996:6). "Los datos económicos: su significado real". Colección: Conocer las claves de la economía. Madrid: Editorial Negocios/Pirámide.

PULIDO, A. (1996:7). "Empleo, inflación, sector público: lo posible y lo imposible. En Colección: Conocer las claves de la economía. Madrid: Editorial Negocios/Pirámide.

PULIDO, A. (1997:1). "El proceso de convergencia y sus consecuencias empresariales". En La empresa española ante la UEM. Círculo de empresarios vascos.

PULIDO, A. (1997:2). "Así entramos en la Comunidad Europea". Colección Economía XXI. Madrid: Editorial Pirámide.

PULIDO, A. (1997:3). “El Big Bang económico”. Colección Economía XXI. Madrid: Editorial Pirámide.

PULIDO, A. (1997:4). "Claves de la economía mundial y española". Colección Economía $X X I$. Madrid: Editorial Pirámide.

PULIDO, A. (1997:5). "En el umbral del siglo XXI. Nuevos mercados, nuevas ideas". Colección Economía XXI. Madrid: Editorial Pirámide.

PULIDO, A. (1998:1). Guía para entender de economía. Madrid: Editorial Pirámide.

PULIDO, A. (1998:2). "Una apuesta por el futuro. Predicciones y profecías económicas". Colección Economía XXI. Madrid: Editorial Pirámide.

PULIDO, A. (1998:3). "En busca de la convergencia europea". Colección Economía XXI. Madrid: Editorial Pirámide.

PULIDO, A. (2000:1). "Crecimiento y nueva economía: Realidades, esperanzas y exageraciones". XIV Reunión Asepelt España, Oviedo 2000.

PULIDO, A. (2000:2). Economía en acción. Madrid: Editorial Pirámide.

PULIDO, A. (2001:1). "Una panorámica de diez años de análisis regional a través del proyecto Hispalink y sus expectativas de futuro". En Pulido y Cabrer, (eds.): Análisis regional: el proyecto Hispalink. pp. 15-21.

PULIDO, A. (2001:2). "Las fuerzas del cambio, La Universidad del siglo XXI: Aspectos a mejorar". Encuentros Multidisciplinares, ISSN-e 1139-9325, Vol. 3, № 8, 2001, pp. 43-47.

PULIDO, A. (2001:3). "El reto de la nueva información TIC". Revista Fuentes Estadísticas, $N^{\circ} 51$, pp. 4-5.

PULIDO, A. (2001:4). "La Nueva Economía: Medición de sus efectos". Información Comercial Española, ICE: Revista de economía, No 793, 2001 (Ejemplar dedicado a: Nuevas tecnologías, ¿nueva economía?), págs. 17-24. 
PULIDO, A. (2001:5). "Precios hedónicos: ajustes por calidad en los precios TIC". Revista Fuentes Estadísticas, $\mathrm{N}^{\circ}$ 58, pp. 5.

PULIDO, A. (2001:6). "Impactos de las nuevas tecnologías en el modelo económico". SEDISI, 25 aniversario.

PULIDO, A. (2001:7). "Consenso de predicciones". Revista Fuentes Estadísticas, № 60, pp. 16.

PULIDO, A. (2001:8). "Fuentes internacionales y nacionales en predicción económica". Revista Fuentes Estadísticas, № 55 , pp. 4-7.

PULIDO, A. (2002:1). "Posibilidades y limitaciones de las Matemáticas en la Economía". Encuentros multidisciplinares. Vol. 4, No 12, 2002 (Ejemplar dedicado a: Nanociencia y nanotecnología: desarrollos actuales y perspectivas), pp. 46-54.

PULIDO, A. (2002:2). "Los Retos y Oportunidades en la Formación y en el Trabajo en la Sociedad de la Información". Federación de Cajas de Ahorros Vasco Navarras.

PULIDO, A. (2002:3). "La Nueva Economía en EE.UU. aún pendiente de la recuperación plena". Revista Fuentes Estadísticas, № 67, pp. 18-19.

PULIDO, A. (2002:4). Viajes por Econolandia. Madrid: Editorial Pirámide.

PULIDO, A. (2003:1). "Mi experiencia como usuario de estadísticas sobre la ampliación". Revista Índice, №1, pp. 11.

PULIDO, A. (2003:2). "Desarrollo sostenible: un reto central para el pensamiento económico". Estudios de Economía Aplicada. Vol.21(2), pp. 203- 220.

PULIDO, A. (2004:1). "Innovación, competitividad y privatización". En Fundación SEPI (ed.): Teoría y política de privatizaciones: su contribución a la modernización económica: análisis del caso español, pp. 185-199.

PULIDO, A. (2004:2). "Innovación, productividad y empleo". Revista universitaria de ciencias del trabajo, $N^{\circ} 5$, pp. 35-44.

PULIDO, A. (2004:3). "Consideraciones para una visión prospectiva de la economía española". Revista Valenciana dÉstudis Autonómics № 45-46, (Ejemplar dedicado a: Globalización y visiones prospectivas), pp. 171-182.

PULIDO, A. (2004:4). "Restricciones medio ambientales al uso de combustibles: limitaciones y mercado de emisiones de CO2". En Martinez-Vall (ed.): La energía en sus claves. IBERDROLA, pp. 858-877.

PULIDO, A. (2005:1). "Los modelos mundiales: un reto pendiente". Revista de economía mundial, № 13, 2005, pp. 15-27.

PULIDO, A. (2005:2). El Sector Financiero en la Comunidad de Madrid. Madrid: Comunidad de Madrid.

PULIDO, A. (2005:3). "Indicadores de calidad en la evaluación del profesorado universitario". Revista Estudios de Economía Aplicada EEA, 23(3), pp. 667-684.

PULIDO, A. (2005:4). "Productividad, competitividad e Innovación". Centre de Recerca Económica (CRE).

PULIDO, A. (2005:5). "Innovación, productividad y empleo". IV Congreso de Castilla y León 2005.

PULIDO, A. (2006:1). "La aventura de la predicción económica y empresarial". En Pulido (ed.): Guía para usuarios de predicciones económicas, pp. 9-30.

PULIDO, A. (2006:2). "Investigación, Innovación y Universidad en la nueva sociedad del conocimiento". Encuentros multidisciplinares, Vol. 8, № 22, 2006 (Ejemplar dedicado a: La investigación y la ciencia en España: perspectivas y propuestas). 
PULIDO, A. (2006:3). "La aventura de la predicción económica y empresarial". Libro 25 aniversario Ceprede.

PULIDO, A. (2006:4). Momentos estelares de Econolandia. Madrid: Ecobook.

PULIDO, A. (2007:1). "Predicción económica: el reto de la globalización". En Cabañes, L. (ed.): Abstracción y realidad en la economía: ensayos en homenaje al profesor Alejandro Lorca Corrons, pp. 373-377.

PULIDO, A. (2007:2). "Reflexiones sobre la universidad del Futuro". IX Foro ANECA, Madrid 2007.

PULIDO, A. (2007:3). "De escenarios de futuro y planes estratégicos para las universidades". Revista Madri+d, $\mathrm{N}^{\circ} 41, \mathrm{pp} .1$.

PULIDO, A. (2007:4). "Conversaciones con Emilio Fontela sobre el futuro de la Universidad". Estudios de Economía Aplicada, 25(3), pp. 591-601. (Ejemplar dedicado a: Mesoeconomía).

PULIDO, A. (2007:5). "Las universidades hacia el 2020: un ejercicio de prospectiva". XXI Reunión ASEPELT Valladolid 2007.

PULIDO, A. (2007:6). "Universidades con futuro: el proyecto UNIVNOVA". Revista Madri+d, $\mathrm{N}^{\circ} 41$, pp.1.

PULIDO, A. (2007:7). "Predicción, Prospectiva, estrategia e innovación: una aplicación a la economía española". En: Prospectiva e Innovación Social.

PULIDO, A. (2007:8). "Cartas a Emilio Fontela". Coordinador. CEPREDE, y Universidad de Nebrija.

PULIDO, A. (2008:1). "Empresa, Innovación y Política Científica". $11^{\circ}$ Congreso de Economía de Castilla y León 2008.

PULIDO, A. (2008:2). "La Innovación en el Siglo XXl”. IBM: Serie de informes, innovación.

PULIDO, A. (2008:3). "Una revisión de conjunto de la economía de los intangibles". Estudios de Economía Aplicada, 26(2), agosto 2008.

PULIDO, A. (2008:4). "Medición del capital intangible a escala macroeconómica y su contribución al crecimiento". XXII Reunión Anual ASEPELT, Barcelona 2008.

PULIDO, A. (2008:5). "Predicción económica: el reto de la globalización". En: Abstracción y Realidad en la Economía. Madrid: Minerva Ediciones.

PULIDO, A. (2008:6). "Relaciones entre economía y medio ambiente". En: Tratado de tributación medioambiental, Vol. 2, 2008, pp. 1075-1087.

PULIDO, A. (2008:7). "El desarrollo Sostenible en el mundo contemporáneo". En: Ética del desarrollo. El mercado y la tecnología. (In Memorian Emilio Fontela)".

PULIDO, A. (2009:1). "Espacio y economía: una combinación con pasado, presente y futuro". Revista de economía ICE. Aspectos territoriales del desarrollo: presente y futuro. Mayo-Junio. $\mathrm{N}^{\circ} 848$, pp. 3-8.

PULIDO, A. (2009:2). "El Futuro de la Universidad". Madrid: Delta publicaciones.

PULIDO, A. (2009:3). "Una comparativa internacional en la medición del capital intangible de países". Revista de Economía Mundial, № 23, pp. 245-262.

PULIDO, A. (2009:4). "La economía en los próximos años: escenarios alternativos". Fundación Sistema Foro sobre Incertidumbres, Retos y Potencialidades del Siglo XXI. Panel sobre 2009.

PULIDO, A. (2010). "La inserción de la economía andaluza en los mercados nacionales e internacionales". En colaboración con: Instituto de Estadística de Andalucía. 
Consejería de Economía, Innovación y Ciencia. IEA-Información Estadística de Andalucía. La transformación de Andalucía 1990-2010. $2^{a}$ época № 1.

PULIDO, A. (2011). "Pepe Barea. Economía y sociedad". En Perez de Uralde y Chaves, (eds.): José Barea Tejeiro: 25 años de CIRIEC-España, pp. 93-94.

PULIDO, A. (2012:1). "Inteligencia económica y empresarial". Presentación en la 63 Junta semestral de predicción de CEPREDE. Córdoba.

PULIDO, A. (2012:2). "España bajo vigilancia: desequilibrios exteriores". Economistas, Año $\mathrm{N}^{\circ} 30, \mathrm{~N}^{\circ}$ Extra 135, pp. 47-55.

PULIDO, A. (2013). "Predicción en tiempos de crisis". Revista de estudios de eCONOMICA, Vol. n 1, pp. 25-36.

PULIDO, A. (2014). "In memoriam Lawrence Robert Klein (1920-2013)". Newsletter International Input-Output Association, Número 25: Febrero, 2014.

PULIDO, A. (2015:1). "Convergencia económica y desigualdad: pasado, presente y futuro". 42 Jornada ASINJA 2015.

PULIDO, A. (2015:2). "La herencia de Klein (1920-2013): Una visión de futuro". Estudios de Economía Aplicada, 33(2), pp. 359-384.

PULIDO, A. (2016:1). "Condicionantes y riesgos internos: Las incertidumbres del momento". Perspectivas económicas y Empresariales, CEPREDE.

PULIDO, A. (2016:2). "Convergencia económica y desigualdad: pasado, presente y futuro". Sistema: Revista de ciencias sociales, № 241 , pp. 35-56.

PULIDO, A. (2016:3). "La economía más allá del 2015". Economistas, № Extra 146-147, (Ejemplar dedicado a: España 2015. Un balance), pp. 175-182.

PULIDO, A. y A. Alcaide (1977). Econometría y modelos econométricos. Madrid: Universidad Nacional de Educación a Distancia.

PULIDO, A. y F. Barbero (1984). "Efectos sobre la economía española de variaciones en el tipo de cambio". Documentos CEPREDE.

PULIDO, A. y B. CABRER (1994). Datos, técnicas y resultados del moderno análisis económico regional. Madrid: Mundiprensa.

PULIDO, A. y J. CALLEALTA (1994). "Situación actual y perspectivas de las regiones de España: XI Jornadas Hispalink" Oviedo 24 y 25 de marzo de 1994.Instituto L. R. Klein.

PULIDO, A. y A. CASTILLA (1982). "A summary of the UAM Econometric Model and Forecast for the Spanish Economy 1982-86". Grupo Link-España, doc. 82/2.

PULIDO, A. y A. CASTILLA (1983). "Wage and Price Equations of the Spanish Econometric Model. Initial Specification". Grupo Link-España, doc. 83/3.

PULIDO, A., R.B. CASTRO y A.M. LÓPEZ (2003). "Hacia una contabilidad regional trimestral sectorial". En Casas y Pulido (eds.): Información económica y técnicas de análisis en el siglo XXI: homenaje al profesor Dr. Jesús B. Pena Trapero, pp. 63-72.

PULIDO, A. y P. FERNÁNDEZ (1986). "Una simulación del sector exterior: integración España-CEE”. Información Comercial Española, № 640. (Ejemplar dedicado a: Primeras Jornadas de Economía Internacional de Valladolid: Comercio Internacional. Política Comercial. CEE), pp. 157-162.

PULIDO, A. y P. FERNÁNDEZ (1990:1). "Experiencias en predicción de la economía española con el modelo Wharton-UAM". Situación: revista de coyuntura económica, $\mathrm{N}^{\circ} .2,1990$, pp. 37-54. 
PULIDO, A. y P. FERNÁNDEZ (1990:2). “Modelización de la demanda del automóvil y los vehículos industriales (Proyecto ANFAC)". Instituto Lawrence R. Klein, Documento 90/DM-1.

PULIDO, A., P. FERNÁNDEZ y E. FONTELA (1987:1). "Exchange rates policies and foreing trade". Instituto Lawrence R. Klein, Documento 87/1.

PULIDO, A., P. FERNÁNDEZ y E. FONTELA (1987:2). "Exchange rates policies and foreing trade: the case of Spain". Association de Econometrie Appliquée.

PULIDO, A. y P. FERNÁNDEZ y J. VICÉNS (1988). "Determinación de precios y salarios en el modelo Wharton-UAM/5". Instituto Lawrence R. Klein, Documento 88/1.

PULIDO, A. y E. FONTELA (1992). "Le modèle Hispalink de l'économie espagnole". En Archambault et Arkhipoff (éds.): La Comptabilité Nationale, pour quoi jaire?. Paris: Económica.

PULIDO, A. y E. FONTELA (1993:1). "Pasado, presente y futuro del análisis input-output". Economía industrial, № 290, pp. 17-24.

PULIDO, A. y E. FONTELA (1993:2). Análisis Input-Output. Modelos, datos y aplicaciones. Madrid: Pirámide.

PULIDO, A. y E. FONTELA (1995). "El papel de las inversiones públicas en las economías industriales avanzadas". Revista de Obras Públicas.

PULIDO, A. y E. FONTELA (2002). "Scenario Models of the World Economy". Cuadernos del Fondo de Investigación Richard Stone, Instituto L.R. Klein. № 7.

PULIDO, A. y E. FONTELA (2004). Principios del Desarrollo Económico Sostenible. Madrid: Fundación Iberdrola.

PULIDO, A. y E. FONTELA (2008). "Innovación y política Científica. Innovación en el Siglo $X X I$ ". IBM: Serie de informes, innovación.

PULIDO, A. E. FONTELA y M. Lo CASCIO (1989). "Productivity surplus distribution: Spanish and Italian results". Instituto Lawrence R. Klein, Documento 89/10.

PULIDO, A., E. FONTELA y J. GUTIÉRREZ (1987). "Etude de prospective du plan bleu de la Mediterranee". INYPSA.

PULIDO, A., E. FONTELA y A.M. del SUR (1988:1). "Enlace de modelos econométricos regionales". Ekonomiaz, № 11 (Ejemplar dedicado a: Análisis Económico Regional), pp. 95-104.

PULIDO, A., E. FONTELA y A.M. del SUR (1988:2). "Linking regional econometric forecasting models". Eighth International Symposium on Forecasting. Regional Forecasting. Amsterdam, The Netherlands.

PULIDO, A. y E. GARCÍA (1986). "Análisis y contrastación del tipo de cambio de la peseta". Instituto Lawrence R. Klein, Documento 86/ME-3.

PULIDO, A. J.V. GARCÍA y G. CORTIÑAS (1964). "Un método de la I.O.: Teoría de grafos". Anales de Economía, №. 5-8, pp. 579-660.

PULIDO, A. y A.M. LÓPEZ (1993). "El sector construcción: aplicación de la metodología input-output al análisis sectorial". Estudios de Economía Aplicada: VII Reunión Anual de ASEPELT-España, Cádiz, 17 y 18 de junio de 1993; Departamento de Economía General, Vol. 3, pp. 128-138.

PULIDO, A. y A.M. LÓPEZ (1999). Predicción y simulación aplicada a la economía y gestión de empresas. Madrid: Pirámide.

PULIDO, A. y A.M. LÓPEZ (2001:1). "Penetración de las TIC y crecimiento económico". Revista del Instituto de Estudios Económicos, № 1-2, pp. 253-272. 
PULIDO, A. y A.M. LÓPEZ (2001:2). "Una propuesta metodológica para evaluar regionalmente la economía digital". XXVII Reunión de Estudios Regionales, Madrid: Noviembre 2001.

PULIDO, A. y A.M. LÓPEZ (2002:1). "Cadenas de producción sectorial y su correspondencia espacial”. XVI Reunión Asepelt, Madrid: 2002.

PULIDO, A. y A.M. LÓPEZ. (2003). "Situación y perspectivas de la Nueva Economía a nivel internacional”. Boletín económico de Andalucía, № 33-34, 2003 (Ejemplar dedicado a: Nueva Economía Y Sociedad del Conocimiento), pp. 51-58.

PULIDO, A., A.M. LÓPEZ y R.B. CASTRO (2003). "La ampliación de la Unión Europea desde la perspectiva regional de España. Análisis del comercio exterior y los flujos de inversión directa". Papeles de economía española, № 103, pp. 187-201.

PULIDO, A., A.M. LÓPEZ y F. RIVERO (1996). "Hispadat, Base de Datos: Hispalink". XV Jornadas, junio 1996: Modelización regional integrada. Instituto de Predicción Económica Lawrence R. Klein.

PULIDO, A. y A. MUR (1993). "Una simulación para el ciclo de la construcción”. Estudios de Economía Aplicada: VII Reunión Anual de ASEPELT-España, Cádiz, 17 y 18 de junio de 1993; Departamento de Economía General, Vol. 1, 1993, pp. 426-437.

PULIDO y otros (1965). "Consideraciones económicas sobre el turismo en España (diversos artículos sobre comercio exterior, empleo y precios)". Estudios Turísticos.

PULIDO y otros (1967:1). "Modelo econométrico de política laboral (1954-71)". Secretaría General Técnica del Ministerio de Trabajo.

PULIDO y otros (1967:2). "Las exportaciones turísticas". Estudios Turísticos, №. 15, pp. 518.

PULIDO y otros (1969). Modelo español de desarrollo educativo. Madrid: Ministerio de Educación.

PULIDO y otros (1970). Modelo econométrico del sector público español. Madrid: Instituto de Estudios Fiscales.

PULIDO y otros (1971). " Modelo COPLAN-I". Comisaria del Plan de desarrollo.

PULIDO y otros (1973:1). Demanda de mano de obra altamente cualificada. Madrid: Dirección General de Universidades e Investigación.

PULIDO y otros (1973:2). Estudio comparativo de las causas que influyen en el desarrollo económico español. Instituto de Desarrollo Económico.

PULIDO y otros (1974). Metodología de desarrollo regional. Madrid: Fundación Juan March.

PULIDO y otros (1975). "La economía del turismo en España". Fondo de Investigación Económica y Social de la Confederación de Cajas de Ahorro.

PULIDO y otros (1976). "Un modelo de previsión del cambio de las formas comerciales". Cuadernos Universitarios de Planificación Empresarial y Marketing, Vol. 2, №. 4, pp. 377-403.

PULIDO y otros (1977:1). "Técnicas de predicción económica alternativa". Cuadernos Universitarios de Planificación Empresarial y Marketing, Vol. 3, №. 3, pp. 245-268.

PULIDO y otros (1977:2). "Financiación de la pequeña y mediana empresa comercial". Instituto de Reformas de las Estructuras Comerciales.

PULIDO y otros (1977:3). "Nuevas formas de comercio en España". Instituto de Reformas de las Estructuras Comerciales. 
PULIDO y otros (1979:1). "Principios básicos para el análisis y predicción de la coyuntura económica desde el punto de vista empresarial". Proyecto de Predicción Económica (PPE), UAM.

PULIDO y otros (1979:2). "Estudio para una reordenación y mejora de la estructura comercial española". Comisión Asesora de Investigación Científica y Técnica.

PULIDO y otros (1980:1). "Reflexiones en torno a un Plan Nacional de Investigación". Dirección General de Política Científica, Cedeti y Fundación Universidad Empresa.

PULIDO y otros (1980:2). "Técnicas de evaluación económica de los aspectos medioambientales". Centro de Estudios de Ordenación del Territorio y Medio Ambiente.

PULIDO y otros (1981:1). "Selección entre proyectos de investigación interdisciplinarios. Algunos antecedentes nacionales e internacionales". Dirección General de Política Científica.

PULIDO y otros (1981:2). "Investigación innovadora". Forum Universidad Empresa Investigación innovadora: Acciones conjuntas Universidad-Empresa dentro de un Plan Nacional de Investigación, pp. 13-18.

PULIDO y otros (1982:1). "Antecedentes del proyecto LINK de modelización económica mundial". Grupo Link-España, documento 82/1.

PULIDO y otros (1982:2). Análisis cuantitativo de la economía española. Modelización de la economía. Madrid: Universidad Autónoma de Madrid.

PULIDO y otros (1982:3). "Especificación inicial de las ecuaciones de consumo". Grupo Link-España, doc. 82/4.

PULIDO y otros (1982:4). "Especificación inicial de las ecuaciones de producción y demanda de factores". Grupo Link-España, documento 82/5.

PULIDO y otros (1983:1). "Proyecto europeo para la elaboración de un modelo macrosectorial multinacional: antecedentes y características básicas (Modelo HERMES)". Grupo CEE-MS España.

PULIDO y otros (1983:2). "Study of a sectorial model for Spain in the context of the Project of a Macrosectorial Multinational Model". Commission of the European Communities, ECl-939-8721.

PULIDO y otros (1983:3). "Especificación inicial de las ecuaciones de comercio exterior". Grupo Link-España, doc. 83/1.

PULIDO y otros (1989:1). "Servicio de seguimiento y predicción a corto plazo: indicadores de alerta". Documentos CEPREDE 1989.

PULIDO y otros (1989:2). "Incorporación de la tecnología en los modelos econométricos: Experiencias con modelos econométricos operativos para predicción y simulaciones de políticas alternativas". Instituto Lawrence R. Klein, Documento 89/3.

PULIDO y otros (2001:1). "Estimación del VAB trimestral de las CC.AA". En Cabrer (ed.): Análisis regional: el proyecto Hispalink, pp. 407-421.

PULIDO y otros (2001:2). "El libro verde de la energía: Una visión española. Conclusiones". En colaboración con: Comisión Europea. Jornada organizada por Club Español de la Energía 2001.

PULIDO y otros (2003). "Balanza comercial con el resto de España". En Mella y Sanz, (eds.): Balanza de Pagos de la Comunidad de Madrid (1998-2000), pp. 71-111.

PULIDO y otros (2007). "Impacto macroeconómico". En: Relevancia de la Biotecnología en España. Madrid: Fundación Genoma España. 
PULIDO y otros (2014). Informe de predicción de la actividad aseguradora en España. Madrid: Fundación Mapfre.

PULIDO, A. y J.B. PENA (1986). "Producción y empleo a largo plazo: una aplicación en la línea Hickman-Coen". Instituto Lawrence R. Klein, Documento 86/ME-2.

PULIDO, A. y J. PÉREZ (1992). "Tratamiento de la deuda pública en el modelo WhartonUAM". Documento 92/6, Instituto L. R. Klein, U.A.M.

PULIDO, A. y J. PÉREZ (2000). "Algunas reflexiones en torno a las técnicas econométricas". Documento de trabajo del Instituto Klein.

PULIDO, A. y J. PÉREZ (2001). Modelos econométricos. Madrid: Ed. Pirámide.

PULIDO, A. y J. PÉREZ (2006:1). "Algunas reflexiones en torno a las técnicas econométricas". En Sur, A. y J. Vicéns (eds.): Estudios en economía: en memoria de Lourdes Barriga Rincón, pp. 263-282.

PULIDO, A. y J. PÉREZ (2006:2). "Las causas del crecimiento económico. Un tema para debate", Estudios de Economía Aplicada, 24(1), pp. 97-103.

PULIDO, A. y J. PÉREZ (2006:3). "Lawrence R. Klein y la economía aplicada". Estudios de Economía Aplicada, 24(1), pp. 43-94.

PULIDO, M.T. y A. PULIDO (1992). "Modelo Micro: modelo simplificado de la Economía Española". Estudios de Economía Aplicada: VI Reunión Anual de ASEPELT-España, 4 y 5 de junio de 1992, Vol. 1, 1992, pp. 3-16.

PULIDO, A. y J.L. ROS (2002). "El entorno económico y empresarial de la Comunidad de Madrid". En "Situación y predicción de la economía madrileña", №. 1, pp. 7-30.

PULIDO, A. y J.L. ROS (2005). "Anexo sobre el proceso de cálculo de las predicciones sectoriales desagregadas". En "Situación y predicción de la economía madrileña", NN. 2, pp. 37-41.

PULIDO, A. y J. SANTOS (1998). Estadística aplicada para ordenadores personales. Madrid: Ed. Pirámide.

PULIDO, A. y A.M. del SUR (1986:1). "Modelo Wharton-UAM/2 de la economía española". Documentos CEPREDE.

PULIDO, A. y A.M. del SUR (1986:2). "Predicción, simulación y análisis de sensibilidad en comercio internacional". Instituto Lawrence R. Klein, Documento 86/3.

PULIDO, A. y A.M. del SUR. (1987). "Experiencias previas en la modelización de la Autonomía de Madrid". Instituto Lawrence R. Klein, Documento 87/ME-5.

PULIDO, A. y J. VICÉNS (1990). "Modelización de la demanda y el consumo del sector de las telecomunicaciones". Instituto Lawrence R. Klein, Documento 90/DM-4.

SAGRISTÁ, A. (1961). "Un estudio preliminar sobre la demanda de aceite de oliva en España". Estadística Española, 12, pp. 14-25.

VIAENE, J.M. (1980). "Custom Union between Spain and E.E.C.: An attempt at quantification of the long term effects in a general equilibrium framework". Disertación doctoral, University of Pennsylvania, Mayo 1980, pp. 42-135.

VICÉNS, J. (1979). "Modelo Econométrico España-Cero". Proyecto de predicción económica, documento 79/8, Universidad Autónoma de Madrid. 